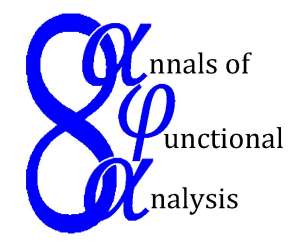

Ann. Funct. Anal. 5 (2014), no. 2, 90-117

$\mathscr{A}$ NNALS OF $\mathscr{F}$ UNCTIONAL $\mathscr{A}$ NALYSIS

ISSN: 2008-8752 (electronic)

URL:www.emis.de/journals/AFA/

\title{
ACTIONS OF ARITHMETIC FUNCTIONS ON MATRICES AND CORRESPONDING REPRESENTATIONS
}

\author{
ILWOO $\mathrm{CHO}^{1 *}$ AND PALLE JORGENSEN ${ }^{2}$ \\ Dedicated to Professor Tsuyoshi Ando \\ Communicated by L. Szekelyhidi
}

\begin{abstract}
In this paper, we study a class of representations of arithmetic functions, and corresponding operator-theoretic and free probabilistic properties. We associate given arithmetic functions $f$ to certain matrices $\alpha_{n}(f)$.
\end{abstract}

\section{INTRODUCTION}

Our aim is to study a harmonic analysis of an algebra $\mathcal{A}$ of arithmetic functions. For this, we introduce a multiplicative convolution on $\mathcal{A}$. While earlier studies of $\mathcal{A}$ have been based on $L$-functions; see (1.1.3) below, we aim here for a more detailed structure. For this we realize representations of $\mathcal{A}$ by $(n \times n)$-matrices $M_{n}(\mathbb{C})$, for all $n \in \mathbb{N}$; as well as representations of $\mathcal{A}$ realized in an infinite tensor product, with tensor factors $M_{n}(\mathbb{C})$. It entails two new elements into the harmonic analysis, (i) Krein spaces, and (ii) free probability spaces. The implications of (i) is that our operators will be acting in certain indefinite inner product spaces. Since we study factorizations, we are naturally led to consideration of independence, but as we note in Sections 4, 5, 6 and 7 below, rather than the traditional notion of independence, we will need the analogous notion of free-independence. The latter being the one arising in consideration of free products and free probability.

Date: Received: October 9, 2013; Accepted: December 1, 2013.

${ }^{*}$ Corresponding author.

2010 Mathematics Subject Classification. Primary 46L53; Secondary 46L54, 47L15, 47L30, 47L55, 05E15, 11G15, 11R04, 11R09, 11R47, 11R56, 46L10, 46L40.

Key words and phrases. Arithmetic function, arithmetic algebra, linear functional, arithmetic prime probability space, Krein space, representation, convolution operator, multiplication operator. 
In [13], we proved that number-theoretic objects derive from operator theory, and operator algebra, via certain representations and free-probability. Motivated by this, we study here some questions in modern number theory and then connect them to operator theory.

One of the direct connection between $p$-adic and Adelic analysis in number theory was considered (e.g., [14]). Independently, arithmetic functions and corresponding Dirichlet series have been studied in an operator-theoretic framework in [12], [15], [16] and [17].

In [16] and [17], the first-named author and Gillespie established a free probabilistic model $\left(\mathcal{A}, \varphi_{x}\right)$ on an algebra $\mathcal{A}$ consisting of all arithmetic functions, using certain linear functionals $\varphi_{x}$, indexed by $x \in \mathbb{R}^{+}$, and containing numbertheoretic computational data.

In [12] and [15], the first-named author studied "truncated" linear functionals $\varphi_{x<y}$. They contain information about primes between two real numbers $x$ and $y$; and corresponding free probabilistic data of arithmetic functions is dictated by these primes. We showed that free distributional data of an arithmetic function $f \in \mathcal{A}$, is completely determined by the primes.

Via our reduction by primes, we realize elements in $\mathcal{A}$ as free random variables. For computations later in our paper, a number of technical results will be needed. They are established in the first half of the paper, and used in the last three sections: Among other things we construct an explicit isomorphism between following two, (i) a certain arithmetic $p$-prime probability space, and (ii) an associated $p$-representation of $\mathcal{A}$ by Krein-space operators under equivalence relations. This in turn allows us (in Section 7) to compute the free joint moments in the two settings. And as a result, we obtain new formulas for these particular free probability spaces, and we give a criterion for when any two given free random variables are identically (free-)distributed.

1.1. Overview. Arithmetic functions are functions $f$ defined from the natural numbers $\mathbb{N}$ into the complex numbers $\mathbb{C}$. In particular, they are tools for constructing (classical) Dirichlet series,

$$
L_{f}(s)=\sum_{k=1}^{\infty} \frac{f(k)}{k^{s}}, \text { for all } s \in \mathbb{C}, \text { for } f \in \mathcal{A} .
$$

$L$-functions also are used in modern number theory; combinatorial number theory, L-function theory, and analytic number theory, etc (e.g., [6], [11], [22], [24], [25], [26] and [35]).

Recall that if $f_{1}, f_{2}$ are arithmetic functions, then the convolution $f_{1} * f_{2}$ is again an arithmetic function, where

$$
f_{1} * f_{2}(n):=\sum_{d \mid n} f_{1}(d) f_{2}\left(\frac{n}{d}\right),
$$

for all $n \in \mathbb{N}$, where " $d \mid n$ " means " $d$ divides $n$," or " $n$ is divisible by $d$," for $d \in \mathbb{N}$. The collection $\mathcal{A}$ of all arithmetic functions forms an algebra, under the usual functional addition and convolution $(*)$.

It is well-known that 
$(1.1 .3)$

$$
\left(L_{f_{1}}(s)\right)\left(L_{f_{2}}(s)\right)=L_{f_{1} * f_{2}}(s) .
$$

We study arithmetic functions as operators in an algebra. So, it is natural to consider representations of arithmetic functions $f$ as certain operators $\Theta_{f}$ acting on a vector space (over the complex numbers $\mathbb{C}$ ).

In [18] and [19], the authors considered representations of $\mathcal{A}$, and found Kreinspace representations for fixed primes. By contrast with usual representation theory, arithmetic functions are understood here as Krein-space operators acting on certain Krein spaces.

We need to emphasize that the Krein-space representations of $\mathcal{A}$ of [18] and [19] are dictated by the choices of primes $p$ and corresponding linear functionals $g_{p}$. To overcome this special condition, we define a new representation in this paper, letting $\mathcal{A}$ act directly on certain (limit of) matricial algebra.

Let $\mathcal{A}$ be the algebra of arithmetic functions as usual, and let $\mathfrak{M}$ be the inductive limit of the matricial algebra $\left\{M_{n}(\mathbb{C})\right\}_{n=1}^{\infty}$.

For any $n \in \mathbb{N}$, define a corresponding subset $D(n)$ of $\mathbb{N}$ by

$$
D(n) \stackrel{\text { def }}{=}\{d \in \mathbb{N}: d \mid n\},
$$

i.e., the set of divisors of $n$, and let

$$
\delta(n) \stackrel{\text { def }}{=}|D(n)|
$$

where $|X|$ means the cardinalities of sets $X$.

For $f \in \mathcal{A}$, and $n \in \mathbb{N}$, define a matrix $\alpha_{n}(f)$ by the $(\delta(n) \times \delta(n))$-lowertriangular matrix

$$
\alpha_{n}(f)=\left(\begin{array}{cccccc}
f(1) & & & & & 0 \\
f\left(d_{2}\right) & f(1) & & & & \\
f\left(d_{3}\right) & f\left(d_{2}\right) & f(1) & & & \\
\vdots & f\left(d_{3}\right) & f\left(d_{2}\right) & f(1) & & \\
\vdots & \ddots & \ddots & \ddots & \ddots & \\
f\left(d_{\delta(n)}\right) & \cdots & \ldots & f\left(d_{3}\right) & f\left(d_{2}\right) & f(1)
\end{array}\right)
$$

in the matricial algebra $M_{\delta(n)}(\mathbb{C})$.

Our operator-algebraic study on arithmetic functions (in terms of free probability) is motivated by the following relation between the multiplication on Dirichlet series of (1.1.1) and the convolution on arithmetic functions defined by (1.1.2), expressed by (1.1.3);

$$
\left(L_{f_{1}}(s)\right)\left(L_{f_{2}}(s)\right)=L_{f_{1} * f_{2}}(s) .
$$

Under our new representation determined by the action $\Theta_{n}$ of (1.1.4), we obtain that:

$$
(\delta(n), 1) \text {-entry of }\left(\alpha_{n}\left(f_{1}\right)\right)\left(\alpha_{n}\left(f_{2}\right)\right)=f_{1} * f_{2}(n)
$$

(See Sections 5 and 6 below).

One can get a system $\left\{\alpha_{n}\right\}_{n=1}^{\infty}$ of morphisms sending arithmetic functions to matrices of $\mathfrak{M}$. Then, for each $n \in \mathbb{N}$, we have a representation $\left(\mathbb{C}^{\oplus \delta(n)}, \alpha_{n}\right)$ of $\mathcal{A}$, 
acting on $\mathfrak{M}$. We study fundamental properties of such representations and we consider operator-theoretic properties of $\alpha_{n}(f)$ 's, for $f \in \mathcal{A}$, and $n \in \mathbb{N}$.

1.2. Technical Backgrounds. In this section, we briefly mention about certain technical backgrounds of this paper. We may not use the following results throughout the paper, but we emphasize they let us have our results meaningful.

As we have seen in (1.1.5), our representations are determined by fixed quantities $n \in \mathbb{N}$, and corresponding numbers $\delta(n)$ of their divisors (See Sections 4 and 5 below). For a sequence $(\delta(n))_{n=1}^{\infty}$, from (1.1.4), we have the following lemma.

Lemma 1.1. Let $\delta(n)$ be in the sense of (1.1.4), for all $n \in \mathbb{N}$.

(1.2.1) For $n \in \mathbb{N}$,

$$
\sum_{k=1}^{n} \delta(k)=n \log n+(2 \gamma-1) n+O(\sqrt{n}),
$$

where $\gamma$ is the Euler constant,

$$
\gamma=\lim _{n \rightarrow \infty}\left(\sum_{k=1}^{n} \frac{1}{k}-\log n\right)=0.57721566 \cdots .
$$

(1.2.2) Let $1_{\mathcal{A}}$ be the arithmetic function, as the (*)-identity element of our arithmetic algebra $\mathcal{A}$, satisfying

$$
1_{\mathcal{A}}(n)= \begin{cases}1 & \text { if } n=1 \\ 0 & \text { otherwise }\end{cases}
$$

for all $n \in \mathbb{N}$. Then

$$
\delta=1_{\mathcal{A}} * 1_{\mathcal{A}}
$$

(1.2.3) Let $f \in \mathcal{A}$. Then

$$
L_{f * 1_{\mathcal{A}}}(s)=L_{f}(s) \zeta(s),
$$

where $\zeta(s)=\sum_{n=1}^{\infty} \frac{1}{n^{s}}$ is the Riemann zeta function.

Proof. The proof of (1.2.1) is done by [33]. The proofs of (1.2.2) and (1.2.3) are done by [16], [17], [18] and [33]

In the rest of this section, we consider relations between our arithmetic algebra $\mathcal{A}$, consisting of all arithmetic functions under the functional addition and convolution, and an algebra

$$
\mathcal{L}=\left\{L_{f}(s): f \in \mathcal{A}\right\}
$$

equipped with the usual functional addition and multiplication.

It is not difficult to check that the set $\mathcal{L}$ is indeed a well-defined algebra, because one can have a linear morphism

$$
L: \mathcal{A} \rightarrow \mathcal{L}
$$

such that

$$
L(f) \stackrel{\text { def }}{=} L_{f} \text { in } \mathcal{L}, \text { for all } f \in \mathcal{A}
$$

By the very definitions (1.2.4) and (1.2.5), one has $L(\mathcal{A})=\mathcal{L}$, and hence, $L$ is surjective, moreover, by (1.2.5), it is injective. Thus, $L$ is a bijective linear transformation from $\mathcal{A}$ onto $\mathcal{L}$. Moreover, by (1.1.3), we have

$$
L\left(f_{1} * f_{2}\right)=L\left(f_{1}\right) L\left(f_{2}\right) \text { in } \mathcal{L},
$$


for all $f_{1}, f_{2} \in \mathcal{A}$. Therefore, the morphism $L$ is an algebra-isomorphism.

Proposition 1.2. Two algebras $\mathcal{A}$ and $\mathcal{L}$ are isomorphic.

The above isomorphic relation of $\mathcal{A}$ and $\mathcal{L}$ is understood as Fourier duality:

$$
f \in \mathcal{A} \longleftrightarrow \widehat{f}(s) \stackrel{\text { def }}{=} L_{f}(s)=\sum_{n=1}^{\infty} \frac{f(n)}{n^{s}} \in \mathcal{L}
$$

For rational simplicity, let $s=\sigma+i t$ in $\mathbb{C}$, i.e., $\operatorname{Re}(\mathrm{s})=\sigma$ and $\operatorname{Im}(\mathrm{s})=t$ in $\mathbb{C}$. Define a linear morphism $M_{B}$ on bounded functions $F$ on $\mathbb{R}$ by

$$
M_{B}(F) \stackrel{\text { def }}{=} \lim _{L \rightarrow \infty} \frac{1}{2 L} \int_{-L}^{L} F(t) d t .
$$

It is called the Bohr mean (e.g., [34]).

Proposition 1.3. Let $f \in \mathcal{A}$, and $\sigma \in \mathbb{R}$. If

$$
t \in \mathbb{R} \longmapsto \widehat{f}(\sigma+i t) \text { is bounded, }
$$

then the following identities hold.

$$
\begin{aligned}
& \sum_{k=1}^{\infty}|f(k)|^{2} k^{-2 \sigma}=M_{B}\left(|\widehat{f}(\sigma+i t)|^{2}\right) ; \\
& f(k) k^{-\sigma}=M_{B}\left(k^{i t} \widehat{f}(\sigma+i t)\right),
\end{aligned}
$$

for all $k \in \mathbb{N}$, and $t \in \mathbb{R}$.

Proof. By applying Bohr harmonic analysis for the bounded function

$$
t \longmapsto \widehat{f}(\sigma+i t),
$$

one can realize that such a bounded function has its almost-periodic Bohr Fourier functions at

$$
\{\log k: k \in \mathbb{N}\} \text {. }
$$

Thus, the identities (1.2.6) and (1.2.7) hold. For more details, see [34], [35] and $[36]$.

\section{PRELiminaries}

In this section, we introduce concepts and theory providing backgrounds of our works.

2.1. Free Probability. We briefly introduce free probability. Free probability is a branch of operator algebra theory, a noncommutative probability theory on noncommutative algebras (e.g., pure algebraic algebras, topological algebras, topological $*$-algebras, etc).

In the free case, we will talk about noncommutative random variables (even if the ambient algebra might be commutative). In classical probability, as is well-known, the work with algebras of random variables are systems of measurable functions on a fixed probability space. The distinction between the two settings, classical vs. algebraic, lies in the meaning of "probability space." In the classical case, it is of interest to focus on systems of independent random variables. But, note that "independence" will then make reference to a given probability space. By contrast, in the algebraic context, i.e., for noncommutative 
random variables, the notion of "probability space" is quite different, and the new property, corresponding to classical independence, is there called "freeness." Its precise definition is recalled in Definition 2.1 below. But to help the reader's intuition, "freeness" is a natural analogue, or extension, of the classical notion of independence.

For free computations, we shall need free cumulants (in the sense of Speicher) playing a major role in combinatorial free probability theory. Speicher's notion is related to the lattice of noncrossing partitions of the set $\{1, \ldots, n\}$, for $n \in \mathbb{N}$, in the same way the more familiar classic functional is computed from the lattice of all partitions of $\{1, \ldots, n\}$.

Definition 2.1. Let $\mathfrak{A}$ be an arbitrary algebra over the complex numbers $\mathbb{C}$, and let $\psi: \mathfrak{A} \rightarrow \mathbb{C}$ be a linear functional on $\mathfrak{A}$. The pair $(\mathfrak{A}, \psi)$ is called a free probability space (over $\mathbb{C})$. All operators $a \in(\mathfrak{A}, \psi)$ are called free random variables (e.g., [34] and [36]).

Note that free probability spaces are depend on the choice of linear functionals.

Definition 2.2. Let $a_{1}, \ldots, a_{s}$ be a free random variable in a $(\mathfrak{A}, \psi)$, for $s \in \mathbb{N}$. The free moments of $a_{1}, \ldots, a_{s}$ are determined by the quantities

$$
\psi\left(a_{i_{1}} \ldots a_{i_{n}}\right),
$$

for all $\left(i_{1}, \ldots, i_{n}\right) \in\{1, \ldots, s\}^{n}$, for all $n \in \mathbb{N}$.

And the free cumulants $k_{n}\left(a_{i_{1}}, \ldots, a_{i_{n}}\right)$ of $a_{1}, \ldots, a_{s}$ is determined by the Möbius inversion;

$$
\begin{aligned}
k_{n}\left(a_{i_{1}}, \ldots, a_{i_{n}}\right) & =\sum_{\pi \in N C(n)} \psi_{\pi}\left(a_{i_{1}}, \ldots, a_{i_{n}}\right) \mu\left(\pi, 1_{n}\right) \\
& =\sum_{\pi \in N C(n)}\left(\prod_{V \in \pi} \psi_{V}\left(a_{i_{1}}, \ldots, a_{i_{n}}\right) \mu\left(0_{|V|}, 1_{|V|}\right)\right),
\end{aligned}
$$

for all $\left(i_{1}, \ldots, i_{n}\right) \in\{1, \ldots, s\}^{n}$, for all $n \in \mathbb{N}$, where $\psi_{\pi}(\ldots)$ means the partitiondepending moments, and $\psi_{V}(\ldots)$ means the block-depending moment.

For example, if

$$
\pi=\{(1,5,7),(2,3,4),(6)\} \text { in } N C(7),
$$

with three blocks $(1,5,7),(2,3,4)$, and $(6)$, then

$$
\begin{aligned}
\psi_{\pi} & \left(a_{i_{1}}^{r_{1}}, \ldots, a_{i_{7}}^{r_{7}}\right) \\
\quad & =\psi(1,5,7)\left(a_{i_{1}}^{r_{1}}, \ldots, a_{i_{7}}^{r_{7}}\right) \psi_{(2,3,4)}\left(a_{i_{1}}^{r_{1}}, \ldots, a_{i_{7}}^{r_{7}}\right) \psi_{(6)}\left(a_{i_{1}}^{r_{1}}, \ldots, a_{i_{7}}^{r_{7}}\right) \\
& =\psi\left(a_{i_{1}}^{r_{1}} a_{i_{5}}^{r_{5}} a_{i_{7}}^{r_{7}}\right) \psi\left(a_{i_{2}}^{r_{2}} a_{i_{3}}^{r_{3}} a_{i_{4}}^{r_{4}}\right) \psi\left(a_{i_{6}}^{r_{6}}\right) .
\end{aligned}
$$

Here, the set $N C(n)$ denotes the noncrossing partition set over $\{1, \ldots, n\}$. It is a lattice with inclusion as $\leq$, such that

$$
\theta \leq \pi \stackrel{\text { def }}{\Longleftrightarrow} \forall V \in \theta, \exists B \in \pi \text {, s.t., } V \subseteq B,
$$

where $V \in \theta$ or $B \in \pi$ means that $V$ is a block of $\theta$, respectively, $B$ is a block of $\pi$, and $\subseteq$ means the usual set inclusion, having its minimal element $0_{n}=\{(1)$, $(2), \ldots,(n)\}$, and its maximal element $1_{n}=\{(1, \ldots, n)\}$.

A partition-dependent free moment $\psi_{\pi}(a, \ldots, a)$ is given by

$$
\psi_{\pi}(a, \ldots, a)=\prod_{V \in \pi} \psi\left(a^{|V|}\right)
$$


where $|V|$ means the cardinality of $V$.

Also, $\mu$ is the Möbius functional from $N C \times N C$ into $\mathbb{C}$, where

$$
N C=\bigcup_{n=1}^{\infty} N C(n) .
$$

i.e., $\mu$ satisfies

$$
\mu(\pi, \theta)=0, \text { for all } \pi>\theta \text { in } N C(n),
$$

and

$$
\mu\left(0_{n}, 1_{n}\right)=(-1)^{n-1} c_{n-1} \text {, and } \sum_{\pi \in N C(n)} \mu\left(\pi, 1_{n}\right)=0,
$$

for all $n \in \mathbb{N}$, where

$$
c_{k}=\frac{1}{k+1}\left(\begin{array}{c}
2 k \\
k
\end{array}\right)=\frac{1}{k+1} \frac{(2 k) !}{k ! k !},
$$

the $k$-th Catalan numbers, for all $k \in \mathbb{N}$. Note that since each $N C(n)$ is a well-defined lattice, if $\pi<\theta$ are given in $N C(n)$, one can decide the "interval"

$$
[\pi, \theta]=\{\delta \in N C(n): \pi \leq \delta \leq \theta\},
$$

and it is always lattice-isomorphic to

$$
[\pi, \theta]=N C(1)^{k_{1}} \times N C(2)^{k_{2}} \times \ldots \times N C(n)^{k_{n}},
$$

for some $k_{1}, \ldots, k_{n} \in \mathbb{N}$, where $N C(l)^{k_{t}}$ means "l blocks of $\pi$ generates $k_{t}$ blocks of $\theta$," for $k_{j} \in\{0,1, \ldots, n\}$, for all $n \in \mathbb{N}$. By the multiplicativity of $\mu$ on $N C(n)$, for all $n \in \mathbb{N}$, if an interval $[\pi, \theta]$ in $N C(n)$ satisfies the above set-product relation, then we have

$$
\mu(\pi, \theta)=\prod_{j=1}^{n} \mu\left(0_{j}, 1_{j}\right)^{k_{j}} .
$$

(For details, see [16], [19] and [34]).

Free moments of free random variables and the free cumulants of them provide equivalent free distributional data. For example, if a free random variable $a$ in $(\mathfrak{A}, \psi)$ is a self-adjoint operator in a von Neumann algebra $\mathfrak{A}$ in the sense that: $a^{*}=a$, then both free moments $\left\{\psi\left(a^{n}\right)\right\}_{n=1}^{\infty}$ and free cumulants $\left\{k_{n}(a, \ldots, a)\right\}_{n=1}^{\infty}$ give the spectral distributional data of $a$.

However, their uses are different. For instance, to study the free distribution of fixed free random variables, the computation of free moments is better; and to study the freeness of distinct free random variables in the structures, the computation and observation of free cumulants is better (e.g., [33] and [34]).

Definition 2.3. We say two subalgebras $A_{1}$ and $A_{2}$ of $\mathfrak{A}$ are free in $(\mathfrak{A}, \psi)$, if all "mixed" free cumulants of $A_{1}$ and $A_{2}$ vanish.. Similarly, two subsets $X_{1}$ and $X_{2}$ of $\mathfrak{A}$ are free in $(\mathfrak{A}, \psi)$, if two subalgebras $A_{1}$ and $A_{2}$, generated by $X_{1}$ and $X_{2}$ respectively, are free in $(\mathfrak{A}, \psi)$. Two free random variables $x_{1}$ and $x_{2}$ are free in $(\mathfrak{A}, \psi)$, if $\left\{x_{1}\right\}$ and $\left\{x_{2}\right\}$ are free in $(\mathfrak{A}, \psi)$.

Suppose $A_{1}$ and $A_{2}$ are free subalgebras in $(\mathfrak{A}, \psi)$. Then the subalgebra $A$ generated both by these free subalgebras $A_{1}$ and $A_{2}$ is denoted by

$$
A:=A_{1} *_{\mathbb{C}} A_{2} .
$$

Assume that $\mathfrak{A}$ is generated by its family $\left\{A_{i}\right\}_{i \in \Lambda}$ of subalgebras, and suppose the subalgebras $A_{i}$ are free from each other in $(\mathfrak{A}, \psi)$, for $i \in \Lambda$. i.e., 


$$
\mathfrak{A}=\underset{i \in \Lambda}{* \mathbb{C}} A_{i} .
$$

Then, we call $\mathfrak{A}$ the free product algebra of $\left\{A_{i}\right\}_{i \in \Lambda}$.

2.2. Indefinite Inner Product Spaces. In this section, we briefly introduce Indefinite inner product spaces and Krein spaces (which are topological indefinite inner product spaces under certain product topology).

Definition 2.4. Let $X$ be a vector space over $\mathbb{C}$ (not necessarily topological), and let [,] be a form on $X$, satisfying that:

$$
[x, y]=\overline{[y, x]} \text {, for all } x, y \in X
$$

and

$$
\begin{aligned}
& {\left[t_{1} x_{1}+t_{2} x_{2}, y_{1}\right]=t_{1}\left[x_{1}, y_{1}\right]+t_{2}\left[x_{2}, y_{1}\right]} \\
& {\left[x_{1}, t_{1} y_{1}+t_{2} y_{2}\right]=t_{1}\left[x_{1}, y_{1}\right]+t_{2}\left[x_{1}, y_{2}\right]}
\end{aligned}
$$

and

$$
[x, x]=0 \text {, whenever } x=0_{X},
$$

for all $t_{1}, t_{2} \in \mathbb{C}$, and $x_{j}, y_{j} \in X$, for $j=1,2$, where $0_{X}$ means the zero vector of $X$.

(2.2.1) If $[x, x] \geq 0$, for all $x \in X$, then we call [,] a positive-definite inner product on $X$.

(2.2.2) If $[x, x] \leq 0$, for all $x \in X$, then [,] is said to be a negative-definite inner product on $X$.

(2.2.3) If neither $[x, x] \geq 0$ nor $[x, x] \leq 0$, for all $x \in X$, then we call [,], an indefinite inner product on $X$.

From the above definition, one can realize that the usual inner products on a vector space $X$ are "positive-definite" inner products on $X$.

Krein spaces are "topological" indefinite inner product spaces characterized by product-topological spaces of Hilbert spaces. Let $H_{j}=\left(H_{j},<,>_{j}\right)$ be Hilbert spaces equipped with their positive-definite inner products $<,>_{j}$, for $j=1,2$. Define the anti-space $\mathrm{H}_{2}^{-}$of $\mathrm{H}_{2}$ by a negative-definite inner product (topological) space,

$$
H_{2}^{-}=\left(H_{2},-<,>_{2}\right),
$$

equipped with the same norm topology with that of $H_{2}$. (So, $H_{2}$ and $H_{2}^{-}$are homeomorphic.) Then the indefinite inner product space $K$,

$$
K=H_{1} \oplus H_{2}^{-},
$$

forms a Krein space, with its indefinite inner product $[,]_{K}$, defined by

$$
\left[\xi_{1} \oplus \eta_{1}, \xi_{2} \oplus \eta_{2}\right]_{K}=<\xi_{1}, \xi_{2}>_{1}+\left(-<\eta_{1}, \eta_{2}>_{2}\right),
$$

equipped with the product topology of topologies of $H_{1}$ and $H_{2}$ (e.g., [1, 2, 3, 4, 5, 21, 27, 29, 32]).

For more about indefinite inner product spaces, see [1, 2, 3, 4, 23]. 


\section{The Inductive Limit Algebra of Matricial Algebras}

For $n \in \mathbb{N}$, let $M_{n}(\mathbb{C})$ be the matricial algebra consisting of all $(n \times n)$-matrices over $\mathbb{C}$. For the system $\left\{M_{n}(\mathbb{C})\right\}_{n=1}^{\infty}$ of matricial algebras, one can define the inductive limit $\mathfrak{M}$ of $\left\{M_{n}(\mathbb{C})\right\}_{n=1}^{\infty}$, as an enveloping algebra, i.e.,

$$
\mathfrak{M} \stackrel{\text { def }}{=} \underset{n}{\lim } M_{n}(\mathbb{C}),
$$

consisting of all $\mathbb{C}$-matrices. Whenever $n_{1}<n_{2}$ in $\mathbb{N}$, an $\left(n_{1} \times n_{1}\right)$-matrix $A$ of $M_{n_{1}}(\mathbb{C})$ is regarded as an $\left(n_{2} \times n_{2}\right)$-matrix, expressed as a form of the block matrix;

$$
\left(\begin{array}{cc}
A & O_{n_{1}}^{n_{2}} \\
O_{n_{2}}^{n_{1}} & O_{n_{2}-n_{1}}
\end{array}\right) \text { in } M_{n_{2}}(\mathbb{C})
$$

in $\mathfrak{M}$, where

$$
\begin{gathered}
O_{n_{2}-n_{1}}=\text { the }\left(\left(n_{2}-n_{1}\right) \times\left(n_{2}-n_{1}\right)\right) \text {-zero matrix } \\
O_{n_{1}}^{n_{2}}=\text { the }\left(n_{1} \times\left(n_{2}-n_{1}\right)\right) \text {-zero matrix, } \\
O_{n_{2}}^{n_{1}}=\text { the }\left(\left(n_{2}-n_{1}\right) \times n_{1}\right) \text {-zero matrix. }
\end{gathered}
$$

Without considering topology, if $A$ is an $(n \times n)$-matrix, then it is understood as the $(\infty \times \infty)$-block matrix,

$$
\left(\begin{array}{ll}
A & O \\
O & O
\end{array}\right)
$$

in the inductive limit $\mathfrak{M}$ of $\left\{M_{n}(\mathbb{C})\right\}_{n=1}^{\infty}$, where $O$ is the $(\infty \times \infty)$-zero matrix.

It is not difficult to check that, if $A \in \mathfrak{M}$, then there exists $N \in \mathbb{N}$, such that $A \in M_{N}(\mathbb{C})$ (possibly, $N \rightarrow \infty$ ).

For $n \in \mathbb{N}$, and $M_{n}(\mathbb{C})$, define morphisms

$$
\pi_{i, j}: M_{n}(\mathbb{C}) \rightarrow \mathbb{C}
$$

by

$$
\pi_{i, j}\left(\left(\begin{array}{cccc}
a_{11} & a_{12} & \cdots & a_{1 n} \\
a_{21} & a_{22} & \cdots & a_{2 n} \\
\vdots & \ddots & \ddots & \vdots \\
a_{n 1} & a_{n 2} & \cdots & a_{n n}
\end{array}\right)\right) \stackrel{\text { def }}{=} a_{i j}
$$

the $(i, j)$-th entries of a matrix, for all $i, j=1, \ldots, n$.

By the very definition of (3.2), each morphism $\pi_{i, j}$ is a linear functional on $M_{n}(\mathbb{C})$. Indeed, if $A_{m}=\left[a_{m: k l}\right]_{k, l} \in M_{n}(\mathbb{C})$, and $t_{m} \in \mathbb{C}$, for $m=1$, 2, then

$$
\begin{aligned}
\pi_{i, j}\left(t_{1} A_{1}\right. & \left.+t_{2} A_{2}\right)=\pi_{i, j}\left(t_{1}\left[a_{1: k l}\right]_{k . l}+t_{2}\left[a_{2: k l}\right]_{k . l}\right) \\
& =\pi_{i, j}\left(\left[t_{1} a_{1: k l}+t_{2} a_{2: k l}\right]_{k, l}\right) \\
& =t_{1} a_{1: i j}+t_{2} a_{2: i j} \\
& =t_{1} \pi_{i, j}\left(\left[a_{1: k l}\right]_{k, l}\right)+t_{2} \pi_{i, j}\left(\left[a_{2: k l}\right]_{k, l}\right) \\
& =t_{1} \pi_{i, j}\left(A_{1}\right)+t_{2} \pi_{i, j} \pi\left(A_{2}\right) .
\end{aligned}
$$

So, the morphisms $\pi_{i, j}$ are linear functionals on $M_{n}(\mathbb{C})$, for all $i, j=1, \ldots, n$, for $n \in \mathbb{N}$. 
In particular, we are interested in the linear functional $\pi_{n, 1}$, for a fixed $n \in \mathbb{N}$. One can get a system $\left\{\pi_{n, 1}\right\}_{n=1}^{\infty}$ of linear functionals.

Define now a linear functional

$$
\pi: \mathfrak{M} \rightarrow \mathbb{C}
$$

by

$$
\pi \stackrel{\text { def }}{=} \underline{\lim } \pi_{n, 1}
$$

in the sense that: if $A \in \mathfrak{M}$, with $A \in M_{N}(\mathbb{C})$, for some $N \in \mathbb{N}$, then

$$
\pi(A) \stackrel{\text { def }}{=} \pi_{N, 1}(A)
$$

where $\pi_{N, 1}$ is in the sense of (3.2).

Definition 3.1. Let $\mathfrak{M}$ be the inductive limit algebra of matricial algebras $\left\{M_{n}(\mathbb{C})\right\}_{n=1}^{\infty}$, and let $\pi$ be the linear functional (3.3) on $\mathfrak{M}$. Then the free probability space $(\mathfrak{M}, \pi)$ in the sense of Section 2 is called the inductive matricial probability space.

Remark 3.2. We will see later, in Section 4, why we are particularly interested in the system $\left\{\pi_{n, 1}\right\}_{n=1}^{\infty}$ of linear functionals.

\section{The ACtions $\alpha_{n}$ of $\mathcal{A}$ Acting on $\mathfrak{M}$}

In this section, we introduce actions of the arithmetic algebra $\mathcal{A}$ on the inductive limit matricial algebra $\mathfrak{M}$. We also understand $\mathfrak{M}$ as the inductive matricial probability space $(\mathfrak{M}, \pi)$, regarded as a system $\left\{M_{n}(\mathbb{C})\right\}_{n=1}^{\infty}$ equipped with the system of linear functionals $\left\{\pi_{n, 1}\right\}_{n=1}^{\infty}$, where $\pi_{n, 1}$ are in the sense of (3.2), for $n$ $\in \mathbb{N}$.

Now, let $n \in \mathbb{N}$. Define a subset $D(n)$ of $\mathbb{N}$ by

$$
D(n) \stackrel{\text { def }}{=}\{d \in \mathbb{N}: d \mid n\},
$$

i.e., $D(n)$ is the set consisting of all divisors of $n$.

Define now a quantity $\delta(n)$ by

$$
\delta(n) \stackrel{\text { def }}{=}|D(n)| \text {, the cardinality of } D(n) .
$$

i.e., $\delta$ is the counting-divisor function in $\mathcal{A}$.

For example, if $p$ is a prime, then

$$
D(p)=\{1, p\} \text { with } \delta(p)=2,
$$

and, generally,

$$
D\left(p^{k}\right)=\left\{1, p, p^{2}, \ldots, p^{k}\right\}
$$

with

$$
\delta\left(p^{k}\right)=k+1
$$

Assumption and Notation Let $n \in \mathbb{N}$ be fixed, and let $D(n)$ be given as in (4.1), with $\delta(n)$-many elements, where $\delta(n)$ is given in (4.2). For convenience, we write 
with the ordering:

$$
D(n)=\left\{d_{1}, d_{2}, \ldots, d_{\delta(n)}\right\}
$$

with

$$
d_{1}<d_{2}<\cdots<d_{\delta(n)} \text { in } \mathbb{N} \text {. }
$$

$$
d_{1}=1, \text { and } d_{\delta(n)}=n .
$$

So, we will write $d_{1}$ and $d_{\delta(n)}$ by 1 and $n$, respectively, for $n \in \mathbb{N}$.

Define now a system $\alpha=\left\{\alpha_{n}\right\}_{n=1}^{\infty}$ of morphisms

$$
\alpha_{n}: \mathcal{A} \rightarrow M_{\delta(n)}(\mathbb{C}) \subset \mathfrak{M},
$$

by

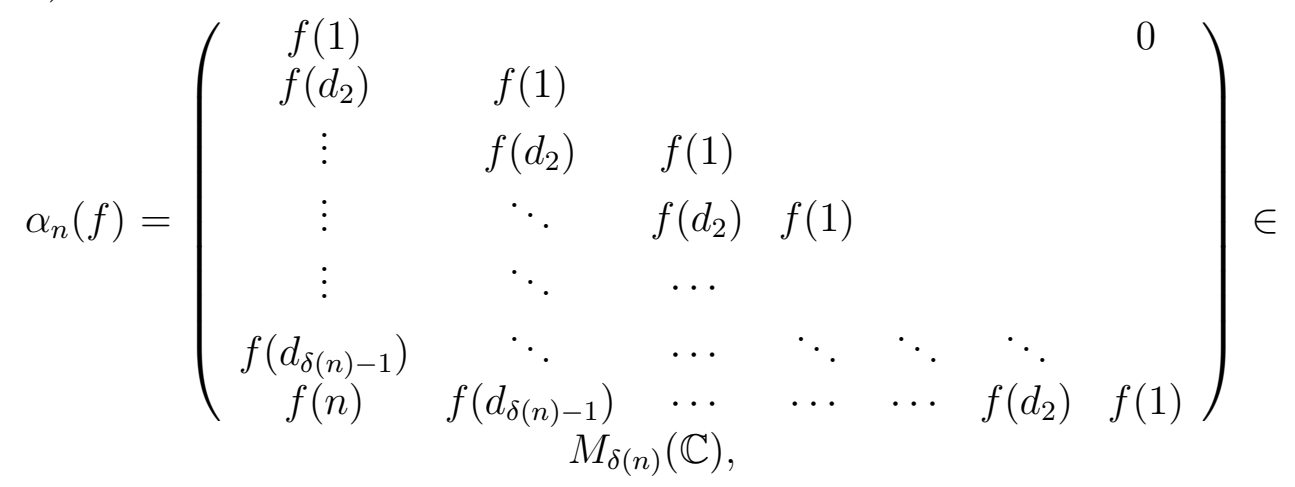

in $\mathfrak{M}$, for all $n \in \mathbb{N}$, (by the notations; $d_{1}=1$, and $d_{\delta(n)}=n$ ), where the right-hand side of (4.3) is a lower-triangular matrix.

It is easy to check that each morphism $\alpha_{n}$ of the system $\alpha=\left\{\alpha_{n}\right\}_{n=1}^{\infty}$ is linear from $\mathcal{A}$ to $M_{\delta(n)}(\mathbb{C})$. i.e., for $f_{1}, f_{2} \in \mathcal{A}$, and $t_{1}, t_{2} \in \mathbb{C}$, one has that:

$$
\alpha_{n}\left(t_{1} f_{1}+t_{2} f_{2}\right)=t_{1} \alpha_{n}\left(f_{1}\right)+t_{2} \alpha_{n}\left(f_{2}\right),
$$

by $(4.3)$.

Theorem 4.1. Let $\alpha=\left\{\alpha_{n}\right\}_{n=1}^{\infty}$ be the system of linear morphisms $\alpha_{n}$ of (4.3). If $f_{1}, f_{2} \in \mathcal{A}$, then

$$
\alpha_{n}\left(f_{1} * f_{2}\right)=\alpha_{n}\left(f_{1}\right) \alpha_{n}\left(f_{2}\right) \text { in } M_{\delta(n)}(\mathbb{C}),
$$

in $\mathfrak{M}$, for all $n \in \mathbb{N}$.

Proof. Let $n$ be a fixed natural number in $\mathbb{N}$, and let $f_{l}$ be arithmetic functions in $\mathcal{A}$, with their corresponding matrices $\alpha_{n}\left(f_{l}\right)$ in $M_{\delta(n)}(\mathbb{C}) \subset \mathfrak{M}$, for $l=1,2$.

If we denote $\alpha_{n}\left(f_{l}\right)$ by $\left[a_{i j}^{(l)}\right]_{\delta(n) \times \delta(n)}$, for $l=1,2$, then

$$
a_{i j}^{(l)}= \begin{cases}0 & \text { if } i<j \\ f_{l}(1) & \text { if } i=j \\ f_{l}\left(d_{k+1}\right) & \text { if } i>j \text { with } i=j+k,\end{cases}
$$

where $k=1,2, \ldots, \delta(n)-1$, for all $l=1,2$. And then one obtains that:

$$
\alpha_{n}\left(f_{1}\right) \alpha_{n}\left(f_{2}\right)=\left[c_{i j}\right]_{\delta(n) \times \delta(n)},
$$


with

$$
c_{i j}=0, \text { whenever } i<j \text { in }\{1,2, \ldots, \delta(n)\},
$$

since the products of lower-triangular matrices are lower-triangular. If $i=j$ in $\{1,2, \ldots, \delta(n)\}$, then it is easy to check that:

$$
c_{i i}=f_{1}(1) f_{2}(1)=\left(f_{1} * f_{2}\right)(1) .
$$

Assume now that $i>j$ with $i=j+k$, for $k=1, \ldots, \delta(n)-1$. Then

by $(4.5)$

$$
\begin{gathered}
c_{j+k, j}=\sum_{s=1}^{\delta(n)} a_{j+k, s}^{(1)} a_{s j}^{(2)} \\
=\sum_{s=0}^{k-1} f_{1}\left(d_{j+k-s}\right) f_{2}\left(d_{j+s}\right)
\end{gathered}
$$

$$
=\sum_{d \mid d_{j+k}} f_{1}(d) f_{2}\left(\frac{d_{j+k}}{d}\right)=f_{1} * f_{2}\left(d_{j+k}\right),
$$

i.e., whenever $i=j+k$ in $\{1, \ldots, \delta(n)\}$, we obtain that

$$
c_{j+k, j}=f_{1} * f_{2}\left(d_{j+k}\right) .
$$

Therefore, by (4.6), (4.7) and (4.8), one has that

$$
\alpha_{n}\left(f_{1}\right) \alpha_{n}\left(f_{2}\right)=\alpha_{n}\left(f_{1} * f_{2}\right),
$$

for $n \in \mathbb{N}$.

By the linearity and by (4.4), the morphism $\alpha_{n}: \mathcal{A} \rightarrow \mathfrak{M}$ is an algebrahomomorphism. Furthermore, since

$$
f_{1} * f_{2}=f_{2} * f_{1} \text { in } \mathcal{A}
$$

one can get that;

$(4.4)^{\prime}$

$$
\alpha_{n}\left(f_{1}\right) \alpha_{n}\left(f_{2}\right)=\alpha_{n}\left(f_{1} * f_{2}\right)=\alpha_{n}\left(f_{2}\right) \alpha_{n}\left(f_{1}\right)
$$

for all $f_{1}, f_{2} \in \mathcal{A}$.

Corollary 4.2. Let $\alpha=\left\{\alpha_{n}\right\}_{n=1}^{\infty}$ be the system of morphisms $\alpha_{n}$ of (4.3), for $n$ $\in \mathbb{N}$. Then each morphism

$$
\alpha_{n}: \mathcal{A} \rightarrow M_{\delta(n)}(\mathbb{C}) \subset \mathfrak{M}
$$

is an algebra-homomorphism.

By the above corollary, we obtain the following proposition.

Proposition 4.3. Let $\alpha$ be the system $\left\{\alpha_{n}\right\}_{n=1}^{\infty}$ of actions $\alpha_{n}$ of $\mathcal{A}$ in the sense of (4.3), for all $n \in \mathbb{N}$. Then this system $\alpha$, itself, is understood as an algebrahomomorphism from the arithmetic algebra $\mathcal{A}$ to the inductive limit algebra $\mathfrak{M}$, satisfying that:

$$
\alpha(f)=\underset{n}{\lim _{\longrightarrow}} \alpha_{n}(f) \text { in } \mathfrak{M}
$$

for all $f \in \mathcal{A}$. 
It shows that, by understanding $\alpha$ as an algebra-homomorphism from $\mathcal{A}$ to $\mathfrak{M}$, we have an algebra dynamical system $(\mathcal{A}, \mathfrak{M}, \alpha)$ of $\mathcal{A}$ acting on $\mathfrak{M}$ via $\alpha$.

Also, by the above proposition, one can obtain the following corollary.

Corollary 4.4. The triple $\left(\mathcal{A}, \mathfrak{M}, \alpha_{n}\right)$ forms an algebra-dynamical system of $\mathcal{A}$ acting on $\mathfrak{M}$, for all $n \in \mathbb{N}$.

Example 4.5. Let's fix $8=2^{3}$ in $\mathbb{N}$. Then

$$
D(8)=\left\{1,2,2^{3}\right\}, \text { with } \delta(8)=3 .
$$

Then, for $f_{l} \in \mathcal{A}$, one has

$$
\alpha_{8}\left(f_{l}\right)=\left(\begin{array}{ccc}
f_{l}(1) & 0 & 0 \\
f_{l}(2) & f_{l}(1) & 0 \\
f_{l}(8) & f_{l}(2) & f_{l}(1)
\end{array}\right) \text { in } M_{3}(\mathbb{C}),
$$

in $\mathfrak{M}$, for $l=1,2$. Also, they satisfy

$$
\begin{aligned}
& \alpha_{8}\left(f_{1}\right) \alpha_{8}\left(f_{2}\right)=\left(\begin{array}{ccc}
f_{1}(1) & 0 & 0 \\
f_{1}(2) & f_{1}(1) & 0 \\
f_{1}(8) & f_{1}(2) & f_{1}(1)
\end{array}\right)\left(\begin{array}{ccc}
f_{2}(1) & 0 & 0 \\
f_{2}(2) & f_{2}(1) & 0 \\
f_{2}(8) & f_{2}(2) & f_{2}(1)
\end{array}\right) \\
& =\left(\begin{array}{ccc}
f_{1}(1) f_{2}(1) & 0 & 0 \\
f_{1}(2) f_{2}(1)+f_{1}(1) f_{2}(2) & f_{1}(1) f_{2}(1) & 0 \\
f_{1}(8) f_{2}(1)+f_{1}(2) f_{2}(2)+f_{1}(1) f_{2}(8) & f_{1}(2) f_{2}(1)+f_{1}(1) f_{2}(2) & f_{1}(1) f_{2}(1)
\end{array}\right) \\
& =\alpha_{8}\left(f_{1} * f_{2}\right) .
\end{aligned}
$$

\section{Free Probabilistic Models $\left\{\left(\mathcal{A}, g_{n}\right)\right\}_{n=1}^{\infty}$}

In this section, we establish a free-probabilistic model for our arithmetic algebra $\mathcal{A}$, with point-evaluations. Moreover, we apply such a model on our dynamical system $(\mathcal{A}, \mathfrak{M}, \alpha)$ of Section 4 .

Recall first that, in [12], [15], [16], [18] and [19], we fix a prime $p$ and constructed the corresponding free-probabilistic model $\left(\mathcal{A}, g_{p}\right)$ and its representations in Krein space. Here, we are interested in our dynamical systems

$$
(\mathcal{A}, \mathfrak{M}, \alpha)=\left\{\left(\mathcal{A}, \mathfrak{M}, \alpha_{n}\right)\right\}_{n=1}^{\infty}=\underset{n}{\underset{\lim }{\longrightarrow}}\left(\mathcal{A}, \mathfrak{M}, \alpha_{n}\right)
$$

of Section 4, and corresponding new free-probabilistic models based on discussions of Section 3. Our new model would be a generalization of those in [12], [15], [16], [18] and [19].

5.1. Free Probability Spaces $\left(\mathcal{A}, g_{n}\right)$ Determined by Point-Evaluations. Let $\mathcal{A}$ be the arithmetic algebra and let $n \in \mathbb{N}$ be a fixed natural number. Similar to $[12],[13],[14],[15],[18]$ and [19], one can define a point-evaluation linear functional $g_{n}$ on $\mathcal{A}$ by

$$
g_{n}(f) \stackrel{\text { def }}{=} f(n) \text {, for all } n \in \mathbb{N} \text {. }
$$


If $n$ is a prime in $\mathbb{N}$, then free probability on the corresponding free probability space $\left(\mathcal{A}, g_{n}\right)$ has been studied in [12], [15] and [16]. In particular, free-moments and free-cumulants of arithmetic functions $f$ of $\mathcal{A}$ are explicitely computed for $g_{n}$, whenever $n$ is a prime. Also, if $n$ is a prime in $\mathbb{N}$, then representations of $\mathcal{A}$, in terns of $g_{n}$, are considered, realized in Krein space in [18] and [19]. The reasons why we restricted our interests to the cases where $n$ are primes in $\mathbb{N}$ were to apply well-known number-theoretic results in our theory.

However, here, we are considering general cases.

Lemma 5.1. For any $n \in \mathbb{N}$, the pairs $\left(\mathcal{A}, g_{n}\right)$ are free probability spaces, where $g_{n}$ are point-evaluations of (5.1.1).

Let $n$ be prime-factorized by

$$
n=p_{1}^{k_{1}} p_{2}^{k_{2}} \cdots p_{m}^{k_{m}}=\Pi_{j=1}^{m} p_{j}^{k_{j}} \text { in } \mathbb{N},
$$

where $p_{1}, \ldots, p_{m}$ are distinct primes, for $m \in \mathbb{N}$, and $k_{1}, \ldots, k_{m} \in \mathbb{N}$.

Clearly, if both $m=1$, and $k_{m}=1$, then we obtain the same results of [12], [15], [16], [18] and [19], for our free-probabilistic models $\left(\mathcal{A}, g_{n}\right)$.

Let's denote $f * \ldots * f(k$-times $)$ by $f^{(k)}$, for all $k \in \mathbb{N}$.

The following proposition shows a relation between free probability spaces $\{(\mathcal{A}$, $\left.\left.g_{n}\right)\right\}_{n=1}^{\infty}$ from the recursive relation (or an algorithm) on free-distributional data of arithmetic functions.

Proposition 5.2. Let $f$ be arithmetic functions in the free probability space $(\mathcal{A}$, $\left.g_{n}\right)$, for a fixed $n \in \mathbb{N}$. Let $D(n)$ be in the sense of (4.1). Then

$$
g_{n}\left(f^{(k+1)}\right)=\sum_{d_{1}, d_{2} \in D(n), n=d_{1} d_{2}} g_{d_{1}}\left(f^{(k)}\right) f\left(d_{2}\right),
$$

for all $k \in \mathbb{N} \cup\{0\}$.

Proof. By convolution,

$$
\begin{aligned}
g_{n}\left(f^{(k+1)}\right) & =g_{n}\left(f^{(k)} * f\right) \\
= & \sum_{d \mid n} f^{(k)}(d) f\left(\frac{n}{d}\right)=\sum_{d \in D(n)} f^{(k)}(d) f\left(\frac{n}{d}\right) \\
= & \sum_{d \in D(n)} g_{d}\left(f^{(k)}\right) f\left(\frac{n}{d}\right),
\end{aligned}
$$

for all $k \in \mathbb{N} \cup\{0\}$.

The above free-moment computation (5.1.3) for $f \in\left(\mathcal{A}, g_{n}\right)$ shows relations between free-moments of $f$ in $\left(\mathcal{A}, g_{n}\right)$, and those of $f$ in $\left\{\left(\mathcal{A}, g_{d}\right)\right\}_{d \in D(n)}$.

In [15], we showed that if $p$ is a prime, then

$$
g_{p}\left(f^{(k)}\right)=k f(1)^{k-1} f(p),
$$

inductively, for all $k \in \mathbb{N}$.

For a prime $p$, since

$$
D\left(p^{2}\right)=\left\{1, p, p^{2}\right\},
$$

one can get that 


$$
g_{p^{2}}\left(f^{(k+1)}\right)=g_{1}\left(f^{(k)}\right) f\left(p^{2}\right)+g_{p}\left(f^{(k)}\right) f(p)+g_{p^{2}}\left(f^{(k)}\right) f(1),
$$

by $(5.1 .3)$, where

$$
g_{1}\left(f^{(k)}\right)=f^{(k)}(1)=(f(1))^{k},
$$

and $g_{p}\left(f^{(k)}\right)$ satisfies (5.1.4), for $k \in \mathbb{N}$, recursively..

Also, with our free-probabilistic language, one can get that:

Proposition 5.3. Let $(\mathcal{A}, \mathfrak{M}, \alpha)$ be the dynamical system in the sense of Section 4. Let $\left\{\left(\mathcal{A}, g_{n}\right)\right\}_{n=1}^{\infty}$ be free probability spaces with $\left\{g_{n}\right\}_{n=1}^{\infty}$ in the sense of (5.1.1). Then we have

$$
\alpha_{n}(f)=\left(\begin{array}{cccccc}
g_{1}(f) & & & & & 0 \\
g_{d_{2}}(f) & g_{1}(f) & & & & \\
\vdots & g_{d_{2}}(f) & g_{1}(f) & & & \\
\vdots & & g_{d_{2}}(f) & g_{1}(f) & & \\
g_{d_{\delta(n)-1}(f)}(f) & \ddots & \ldots & \ddots & \ddots & \\
g_{n}(f) & g_{d_{\delta(n)-1}}(f) & \ldots & \ldots & g_{d_{2}}(f) & g_{1}(f)
\end{array}\right)
$$

The above proposition shows the relation between the dynamical systems $\{(\mathcal{A}$, $\left.\left.\mathfrak{M}, \alpha_{n}\right)\right\}_{n=1}^{\infty}$ and the free probability spaces $\left\{\left(\mathcal{A}, g_{n}\right)\right\}_{n=1}^{\infty}$.

5.2. Free-Homomorphic Relation. In this section, motivated by (5.1.5), we study refined relations between free probability spaces $\left\{\left(\mathcal{A}, g_{n}\right)\right\}_{n=1}^{\infty}$ and dynamical systems $\left\{\left(\mathcal{A}, \mathfrak{M}, \alpha_{n}\right)\right\}_{n=1}^{\infty}$, more in detail. Recall linear functionals $\pi=$ $\left\{\pi_{n, 1}\right\}_{n=1}^{\infty}$ of (3.3) on $\mathfrak{M}$, where each $\pi_{n, 1}$ is in the sense of $(3.2)$, for all $n \in \mathbb{N}$.

By definition, one can get that:

Lemma 5.4. Let $L T_{n}$ be the subset of $M_{n}(\mathbb{C})$ consisting of all lower-triangular matrices, for all $n \in \mathbb{N}$. Then $\alpha_{n}(\mathcal{A})$ is homomorphic to $L T_{\delta(n)}$, for all $n \in \mathbb{N}$.

Proof. By definition, $\alpha_{n}(\mathcal{A})$ is a subset of $L T_{\delta(n)}$, for all $n \in \mathbb{N}$. Since each morphism $\alpha_{n}$ is an algebra-homomorphism from $\mathcal{A}$ into $M_{\delta(n)}(\mathbb{C})$, it is sufficient to show that $L T_{\delta(n)}$ is a subalgebra of $M_{\delta(n)}(\mathbb{C})$, but it is trivial, because the matricial sums and the matricial multiplications of lower-triangular matrices are lower-triangular, too.

By the above lemma, the algebra $\alpha_{n}(\mathcal{A})$ is a subalgebra of $L T_{\delta(n)}$, in $M_{\delta(n)}(\mathbb{C})$, for each $n \in \mathbb{N}$. Thus, we can restrict the linear functional $\pi_{\delta(n), 1}$ on $\alpha_{n}(\mathcal{A})$, for all $n \in \mathbb{N}$. In other words, we obtain free probability spaces

$$
\left\{\left(\alpha_{n}(\mathcal{A}), \pi_{\delta(n), 1}\right)\right\}_{n=1}^{\infty} .
$$

Definition 5.5. Two arbitrary free probability spaces $\left(A_{1}, \varphi_{1}\right)$ and $\left(A_{2}, \varphi_{2}\right)$ are said to be free-homomorphic, if (i) there exists an algebra-homomorphism

$$
h: A_{1} \rightarrow A_{2},
$$

and (ii) $\varphi_{2}(h(a))=\varphi_{1}(a)$, for all $a \in A_{1}$. 
For example, if a homomorphism $h$ of the above definition is an "algebraisomorphism," then our free-homomorphic relation is nothing but the equivalence (in the sense of Voiculescu, e.g., see [34] and [36]) of free probability spaces.

Theorem 5.6. The free probability space $\left(\mathcal{A}, g_{n}\right)$ is free-homomorphic to the free probability space $\left(\alpha_{n}(\mathcal{A}), \pi_{\delta(n), 1}\right)$, for all $n \in \mathbb{N}$. Furthermore, for all $f \in \mathcal{A}$, one has

$$
\pi_{\delta(n), 1}\left(\alpha_{n}(f)\right)=g_{n}(f)
$$

for all $n \in \mathbb{N}$.

Proof. Let's fix $n \in \mathbb{N}$. By the above lemma, there exists an algebra-homomorphism $\alpha_{n}$ from $\mathcal{A}$ into $\alpha_{n}(\mathcal{A})$ in $L T_{\delta(n)}$. Thus it suffice to check that

$$
\pi_{\delta(n), 1}\left(\alpha_{n}(f)\right)=g_{n}(f),
$$

for all $f \in \mathcal{A}$. However, it is trivial by (5.1.5).

By the above free-homomorphic relation between

$$
\left(\mathcal{A}, g_{n}\right) \text { and }\left(\alpha_{n}(\mathcal{A}), \pi_{\delta(n), 1}\right)
$$

the study of free-structures of $\mathcal{A}$ in terms of $g_{n}$ is to investigate those of $\alpha_{n}(\mathcal{A})$ in terms of $\pi_{\delta(n), 1}$.

Of course, if $n$ is prime, then the results of [12], [15], [16], [18] and [19] cover the theory. Thus, we are interested in the cases where $n$ is not a prime.

Mimicking the above theorem, we can get the following general results for free-homomorphic relations.

Corollary 5.7. Let $\left\{\left(\mathcal{A}, g_{n}\right)\right\}_{n=1}^{\infty}$ be free probability spaces of $\mathcal{A}$ determined by point-evaluations. For a fixed $n \in \mathbb{N}$, with

$$
D(n)=\left\{d_{1}=1<d_{2}<\cdots<d_{\delta(n)}=n\right\},
$$

we have

$$
\pi_{i, j}\left(\alpha_{n}(f)\right)=g_{d_{i-j+1}}(f)=f\left(d_{i-j+1}\right),
$$

whenever $i \geq j$, and

$$
\pi_{i, j}\left(\alpha_{n}(f)\right)=0 \text {, whenever } i<j,
$$

where $\pi_{i j}$ are in the sense of (3.2) and (3.3), for all $i, j \in\{1, \ldots, \delta(n)\}$.

5.3. The Cases where $n=p^{k}$. In this section, we restrict our interests to the cases where a fixed natural number $n$ is a form of $p^{k}$, where $p$ is a prime, and $k \in$ $\mathbb{N}$. Notice that, if $k=1$, equivalently, if $n=p$, then the theory of [12], [15], [16], [18] and [19] is applicable. Thus we may further restrict our interests to where $k$ $>1$.

Now, fix $p^{k} \in \mathbb{N}$, where $p$ is a prime and $k \in \mathbb{N}$. Then

$$
D\left(p^{k}\right)=\left\{1, p, p^{2}, \ldots, p^{k}\right\}
$$

with

$$
\delta\left(p^{k}\right)=k+1 .
$$


As we have seen in Sections 5.1 and 5.2, the corresponding free probability space $\left(\mathcal{A}, g_{p^{k}}\right)$ of the arithmetic algebra $\mathcal{A}$ is free-homomorphic to $\left(\alpha_{p^{k}}(\mathcal{A}), \pi_{k+1,1}\right)$. So, one can get that:

Corollary 5.8. Let $f \in\left(\mathcal{A}, g_{p^{k}}\right)$ with its corresponding matrix $\alpha_{p^{k}}(f)$ in $L T_{k+1}$. Then

$$
\alpha_{p^{k}}(f)=\left[c_{i j}\right]_{(k+1) \times(k+1)} \text { in } L T_{k+1}
$$

with

$$
c_{i j}= \begin{cases}0 & \text { if } i<j \\ g_{1}(f)=f(1) & \text { if } i=j \\ g_{p^{i-j}}(f)=f\left(p^{i-j}\right) & \text { if } i>j\end{cases}
$$

for all $i, j \in\{1, \ldots, k+1\}$.

Proof. The proof of (5.3.1) is done by (5.2.1).

Now, let $\alpha_{p^{k}}(f)$ be the matrix in $L T_{k+1}$ as in (5.3.1). Consider the uppercornered blocks $\alpha_{p^{k}: j}(f)$ of $\alpha_{p^{k}}(f)$, for $j=1, \ldots, k+1=\delta\left(p^{k}\right)$;

$$
\begin{aligned}
& \alpha_{p^{k}: 1}(f)=[f(1)]=f(1), \\
& \alpha_{p^{k}: 2}(f)=\left(\begin{array}{cc}
f(1) & 0 \\
f(p) & f(1)
\end{array}\right), \\
& \alpha_{p^{k}: 3}(f)=\left(\begin{array}{ccc}
f(1) & 0 & 0 \\
f(p) & f(1) & 0 \\
f\left(p^{2}\right) & f(p) & f(1)
\end{array}\right), \\
& \alpha_{p^{k}: k+1}(f)=\alpha_{p^{k}}(f) .
\end{aligned}
$$

It is not hard to check that the block matrix $\alpha_{p^{k}: 2}(f)$ of $\alpha_{p^{k}}(f)$ is understood as operators $\Theta_{f}$ in the sense of [18] and [19].

Different from the arbitrary cases for $n \in \mathbb{N}$, if $n$ has its form as $p^{k}$, for a prime $p$ for some $k \in \mathbb{N}$, then we obtain the following block-matricial information.

Theorem 5.9. Let $p^{k}$ be fixed as above, and let $f$ be a free random variable of $\left(\mathcal{A}, g_{p^{k}}\right)$ with its corresponding matrix $\alpha_{p^{k}}(f)$ in $L T_{k+1}$. The upper-cornered block matrices

$$
\left\{\alpha_{p^{k}: j}(f)\right\}_{j=1}^{k+1} \text { of } \alpha_{p^{k}}(f)
$$

in the sense of (5.3.2) are identical to the realized matrices $\alpha_{p^{j-1}}(f)$ in $L T_{j}$, for all $j=1, \ldots, k+1$. i.e.,

$$
\alpha_{p^{k}: j}(f)=\alpha_{p^{j-1}}(f), \text { for } j=1, \ldots, k+1 .
$$

Proof. The proof is inductively done by (5.3.1) and by the definition (5.3.2). 
As we mentioned above, such a recursive relation (5.3.3) dose not hold in general for arbitrary $n \in \mathbb{N}$. Also, the relation (5.3.3) demonstrates that the freeprobabilistic data of $f$ in $\left(\mathcal{A}, p^{k}\right)$ contain those of $f$ in $\left\{\left(\mathcal{A}, p^{j}\right)\right\}_{j=1}^{k}$, under our representations $\left\{\alpha_{p^{j}}\right\}_{j=1}^{k}$.

Define now projections $\left\{P_{j}\right\}_{j=1}^{\infty}$ in $\mathfrak{M}$ by

$$
\begin{aligned}
& P_{1}=\left(\begin{array}{cccc}
1 & & & 0 \\
& 0 & & \\
& & 0 & \\
0 & & & \ddots
\end{array}\right) \\
& P_{2}=\left(\begin{array}{ccccc}
1 & & & & 0 \\
& 1 & & & \\
& & 0 & & \\
& & & 0 & \\
0 & & & & \ddots
\end{array}\right)
\end{aligned}
$$

$\cdots$

$$
P_{3}=\left(\begin{array}{ccccc}
1 & & & & \\
& 1 & & & \\
& & 1 & & \\
& & & 0 & \\
& & & & \ddots
\end{array}\right)
$$

and

$$
P_{k}=I_{k} \oplus O \text {, the block matrix in } \mathfrak{M} \text {. }
$$

Then, by (5.3.3),

$$
\alpha_{p^{j}}(f)=\left(P_{j-1}\right)\left(\alpha_{p^{k}}(f)\right) \text { in } \mathfrak{M},
$$

for $j=1,2, \ldots, k-1$, for all $k \in \mathbb{N}$.

Corollary 5.10. Let $f \in \mathcal{A}$ be an arithmetic function. For a prime $p$ and $k \in \mathbb{N}$, let $\alpha_{p^{k}}(f)$ be the realized matrix of $f$ in $L P_{k+1}$ in $\mathfrak{M}$, under the dynamical system $\left(\mathcal{A}, \mathfrak{M}, \alpha_{p^{k}}\right)$. Understand $f$ as free random variables in free probability spaces $(\mathcal{A}$, $\left.g_{p^{j}}\right)$, for $j=0,1,2, \ldots, k$. Then

$$
g_{p^{j}}(f)=\pi_{j+1,1}\left(P_{j}\left(\alpha_{p^{k}}(f)\right)\right),
$$

for all $j=0,1, \ldots, k$, where $\left\{P_{j}\right\}_{j=1}^{k}$ are in the sense of (5.3.4).

Proof. The proof of (5.3.5) is done by (5.2.1), (5.3.2) and (5.3.4). 


\section{Representations of $\mathcal{A}$ in Indefinite Inner Product Spaces}

In this section, we study representations of the arithmetic algebra $\mathcal{A}$ for a fixed natural number $p^{k}$, where $p$ is a prime and $k \in \mathbb{N}$. Throughout this section, we fix a prime $p$ and $k$ in $\mathbb{N}$.

Define first a relation $\mathcal{R}_{p^{k}}$ on $\mathcal{A}$ by

$$
f_{1} \mathcal{R}_{p^{k}} f_{2} \stackrel{\text { def }}{\Longleftrightarrow} f_{1}\left(p^{j}\right)=f_{2}\left(p^{j}\right),
$$

for all $j=0,1, \ldots, k$.

Then the relation $\mathcal{R}_{p^{k}}$ on $\mathcal{A}$ is an equivalence relation. Indeed, it satisfies that: $f_{1} \mathcal{R}_{p^{k}} f_{1}$, for all $f \in \mathcal{A}$,

$f_{1} \mathcal{R}_{p^{k}} f_{2} \Longrightarrow f_{2} \mathcal{R}_{p^{k}} f_{1}$, for all $f_{1}, f_{2} \in \mathcal{A}$, and

$f_{1} \mathcal{R}_{p^{k}} f_{2}$ and $f_{2} \mathcal{R}_{p^{k}} f_{3} \Longrightarrow f_{1} \mathcal{R}_{p^{k}} f_{3}$

for all $f_{1}, f_{2}, f_{3} \in \mathcal{A}$.

Construct now the quotient algebra

$$
\mathcal{A}_{p^{k}} \stackrel{\text { def }}{=} \mathcal{A} / \mathcal{R}_{p^{k}}
$$

of $\mathcal{A}$, induced by the equivalence relation $\mathcal{R}_{p^{k}}$ of (6.1).

Let $\mathbb{C}^{k+1}$ be the $(k+1)$-dimensional vector space over $\mathbb{C}$. In $\mathbb{C}^{k+1}$, define a subset $\mathcal{V}_{p^{k}}$ by

$$
\mathcal{V}_{p^{k}}=\left\{\left(f(1), f(p), f\left(p^{2}\right), \ldots, f\left(p^{k}\right)\right): f \in \mathcal{A}_{p^{k}}\right\},
$$

where $\mathcal{A}_{p^{k}}$ is in the sense of (6.2).

It is not difficult to verify that this subset $\mathcal{V}_{p^{k}}$, induced by $\mathcal{A}_{p^{k}}$, is a subspace of $\mathbb{C}^{k+1}$. Indeed, if we take two elements $v_{1}$ and $v_{2}$ in $\mathcal{V}_{p^{k}}$, then one can understand them as

$$
v_{j}=\left(f_{j}(1), f_{j}(p), f_{j}\left(p^{2}\right), \ldots, f_{j}\left(p^{k}\right)\right),
$$

for $j=1,2$, for some $f_{1}, f_{2} \in \mathcal{A}$. So, if $t_{1}, t_{2} \in \mathbb{C}$, then

$$
\begin{aligned}
t_{1} v_{1} & +t_{2} v_{2} \\
& =\left(\left(t_{1} f_{1}+t_{2} f_{2}\right)(1),\left(t_{1} f_{1}+t_{2} f_{2}\right)(p), \ldots,\left(t_{1} f_{1}+t_{2} f_{2}\right)\left(p^{k}\right)\right),
\end{aligned}
$$

also contained in $\mathcal{V}$, because $t_{1} f_{1}+t_{2} f_{2}$ is an arithmetic function in $\mathcal{A}$, too. i.e., whenever $v_{1}, v_{2} \in \mathcal{V}_{p^{k}}$, and $t_{1}, t_{2} \in \mathbb{C}$, then

$$
t_{1} v_{1}+t_{2} v_{2} \in \mathcal{V}_{p^{k}}, \text { too. }
$$

Proposition 6.1. The subset $\mathcal{V}_{p^{k}}$ of $\mathbb{C}^{k+1}$ induced by the quotient algebra $\mathcal{A}_{p^{k}}$ of the arithmetic algebra $\mathcal{A}$ is a subspace of $\mathbb{C}^{k+1}$, for all $k \in \mathbb{N}$.

Proof. The proof is done by the discussion of the very above paragraph.

We call $\mathcal{V}_{p^{k}}$, the arithmetic(-functional) subspace of $\mathbb{C}^{k+1}\left(\right.$ for $\left.p^{k}\right)$.

Let $A_{o}$ be a matrix in $M_{k+1}(\mathbb{C})$, defined by

$$
A_{o}=\left[a_{i j}\right]_{(k+1) \times(k+1)},
$$

with 


$$
a_{i j}= \begin{cases}1 & \text { if } i+j=k+1 \\ 0 & \text { otherwise }\end{cases}
$$

i.e., it is a matrix whose skew-diagonal entries are 1, and all other entries are 0 .

On the arithmetic subspace $\mathcal{V}_{p^{k}}$ of $\mathbb{C}^{k+1}$, define a morphism

$$
[,]_{p^{k}}: \mathcal{V}_{p^{k}} \times \mathcal{V}_{p^{k}} \rightarrow \mathbb{C}
$$

by

$$
\begin{aligned}
{\left[\left(f_{1}(1),\right.\right.} & \left.\left.f_{1}(p), \ldots, f_{1}\left(p^{k}\right)\right),\left(f_{2}(1), f_{2}(p), \ldots, f_{2}\left(p^{k}\right)\right)\right]_{p^{k}} \\
& \stackrel{\operatorname{def}}{=}\left(\begin{array}{c}
f_{1}(1) \\
f_{1}(p) \\
\vdots \\
f_{1}\left(p^{k}\right)
\end{array}\right) \bullet A_{o}\left(\begin{array}{c}
\overline{f_{2}(1)} \\
\frac{f_{2}(p)}{\vdots} \\
\overline{f_{2}\left(p^{k}\right)}
\end{array}\right) \\
& =f_{1}(1) \overline{f_{2}\left(p^{k}\right)}+f_{1}(p) \overline{f_{2}\left(p^{k-1}\right)}+\ldots+f_{1}\left(p^{k}\right) \overline{f_{2}(1)} \\
& =\sum_{j=0}^{k} f_{1}\left(p^{j}\right) \overline{f_{2}\left(p^{k-j}\right)}
\end{aligned}
$$

where $A_{o}$ is in the sense of $(6.4)$, and where $(\bullet)$ means the usual dot product on $\mathbb{C}^{k+1}$. Here, $\bar{z}$ means the conjugate of $z$, for all $z \in \mathbb{C}$.

Then, since $A_{o}$ is self-adjoint in the sense that: its adjoint $A_{o}^{*}$ and itself $A_{o}$ are identical in $M_{k+1}(\mathbb{C})$, and since the dot product $(\bullet)$ is sesqui-linear, the morphism $[,]_{p^{k}}$ on $\mathcal{V}$ is sesqui-linear, too.

Lemma 6.2. The morphism $[,]_{p^{k}}$ of (6.5) on the arithmetic subspace $\mathcal{V}_{p^{k}}$ of $\mathbb{C}^{k+1}$ is a sesqui-linear form. i.e., one has

(6.6)

$$
\left[t_{1} v_{1}+t_{2} v_{2}, v_{3}\right]_{p^{k}}=t_{1}\left[v_{1}, v_{3}\right]_{p^{k}}+t_{2}\left[v_{2}, v_{3}\right]_{p^{k}}
$$

and

$$
\left[v_{1}, t_{2} v_{2}+t_{3} v_{3}\right]_{p^{k}}=\overline{t_{2}}\left[v_{1}, v_{2}\right]_{p^{k}}+\overline{t_{3}}\left[v_{1}, v_{3}\right]_{p^{k}}
$$

for all $v_{j} \in \mathcal{V}_{p^{k}}, t_{j} \in \mathbb{C}$, for $j=1,2,3$.

Proof. The proof is done by the very definition (6.5).

Moreover, this sesqui-linear form $[,]_{p^{k}}$ is an indefinite inner product on $\mathcal{V}_{p^{k}}$. Indeed, the for $[,]_{p^{k}}$ is sesqui-linear by the above proposition, and it also satisfies that:

$$
[v, v]_{p^{k}}=0 \Longrightarrow v=0_{p^{k}} \text { in } \mathcal{V}_{p^{k}}
$$

where $0_{p^{k}}$ means the zero vector of $\mathcal{V}_{p^{k}}$. We need to be careful here that the arithmetic subspace $\mathcal{V}_{p^{k}}$ of $\mathbb{C}^{k+1}$ is induced by the quotient algebra $\mathcal{A}_{p^{k}}=\mathcal{A} /$ $\mathcal{R}_{p^{k}}$, not by $\mathcal{A}$. Therefore, the relation (6.7) holds.

And the form $[,]_{p^{k}}$ satisfies that: 
by $(6.5)$

$$
\begin{gathered}
{\left[\left(f(1), f(p), \ldots, f\left(p^{k}\right)\right),\left(f(1), f(p), \ldots, f\left(p^{k}\right)\right)\right]_{p^{k}}} \\
=\sum_{j=0}^{k} f\left(p^{j}\right) \overline{f\left(p^{k-j}\right)}
\end{gathered}
$$

contained in the real numbers $\mathbb{R}$, i.e.,

$$
\begin{aligned}
& =\frac{1}{2} \sum_{j=0}^{k}\left(f\left(p^{j}\right) \overline{f\left(p^{k-j}\right)}+f\left(p^{k-j}\right) \overline{f\left(p^{j}\right)}\right) \\
& =\frac{1}{2} \sum_{j=0}^{k} 2 \operatorname{Re}\left(\mathrm{f}\left(\mathrm{p}^{\mathrm{j}}\right) \overline{\mathrm{f}\left(\mathrm{p}^{\mathrm{k}-\mathrm{j}}\right)}\right) \\
& =\sum_{j=0}^{k} \operatorname{Re}\left(\mathrm{f}\left(\mathrm{p}^{\mathrm{j}}\right) \overline{\mathrm{f}\left(\mathrm{p}^{\mathrm{j}-\mathrm{j}}\right)}\right)
\end{aligned}
$$

$$
[v, v]_{p^{k}} \in \mathbb{R} \text {, for all } v \in \mathcal{V}_{p^{k}} .
$$

Also, one can easily get that:

$$
\left[v_{1}, v_{2}\right]_{p^{k}}=\overline{\left[v_{2}, v_{1}\right]_{p^{k}}}, \text { for all } v_{1}, v_{2} \in \mathcal{V}_{p^{k}}
$$

Therefore, by (6.6) through (6.9), we obtain the following proposition.

Proposition 6.3. Let $[,]_{p^{k}}$ be the sesqui-linear form (6.5) on the arithmetic subspace $\mathcal{V}_{p^{k}}$ of $\mathbb{C}^{k+1}$. Then it is an indefinite inner product on $\mathcal{V}_{p^{k}}$.

Proof. The proof is directly followed from (6.6), (6.7), (6.8) and (6.9), by Section 2.2 .

So, we obtain an indefinite inner product space $\left(\mathcal{V}_{p^{k}},[,]_{p^{k}}\right)$. If there is no confusion, we keep writing the indefinite inner product space simply by $\mathcal{V}_{p^{k}}$.

Definition 6.4. We call $\mathcal{V}_{p^{k}}=\left(\mathcal{V}_{p^{k}},[,]_{p^{k}}\right)$, the arithmetic indefinite inner product subspace of $\mathbb{C}^{k+1}$.

Now, observe $[,]_{p^{k}}$ under our language;

by (6.5)

$$
\begin{gathered}
{\left[\left(f_{1}(1), f_{1}(p), \ldots, f_{1}\left(p^{k}\right)\right),\left(f_{2}(1), f_{2}(p), \ldots, f_{2}\left(p^{k}\right)\right)\right]_{p^{k}}} \\
=\sum_{j=0}^{k} f_{1}\left(p^{j}\right) \frac{f_{2}\left(p^{k-j}\right)}{}
\end{gathered}
$$

$$
\begin{aligned}
& =\sum_{d \in D\left(p^{k}\right)} f_{1}(d) \overline{f_{2}\left(\frac{p^{k}}{d}\right)} \\
& =\left(f_{1} * f_{2}^{*}\right)\left(p^{k}\right),
\end{aligned}
$$

where $h^{*}$ means the arithmetic function in $\mathcal{A}$ (or in $\mathcal{A}_{p^{k}}$ ), induced by $h$ in $\mathcal{A}$ (respectively, in $\mathcal{A}_{p^{k}}$ ) such that

$$
h^{*}(n) \stackrel{\text { def }}{=} \overline{h(n)} \text {, for all } n \in \mathbb{N} .
$$

i.e., one obtains the following relation.

Theorem 6.5. Let $\mathcal{V}_{p^{k}}$ be the arithmetic indefinite inner product subspace of $\mathbb{C}^{k+1}$, and let

for $j=1,2$. Then

$$
v_{j}=\left(f_{j}(1), f_{j}(p), \ldots, f_{j}\left(p^{k}\right)\right) \in \mathcal{V}_{p^{k}},
$$

(6.11) 


$$
\left[v_{1}, v_{2}\right]_{p^{k}}=\left(f_{1} * f_{2}^{*}\right)\left(p^{k}\right)=g_{p^{k}}\left(f_{1} * f_{2}^{*}\right) .
$$

Proof. The proof of (6.11) is from (6.10).

The above theorem let us understand there are relations between our free probability spaces $\left(\mathcal{A}, g_{p^{k}}\right)$ and the arithmetic indefinite inner product subspaces $\mathcal{V}_{p^{k}}$ of $\mathbb{C}^{k+1}$, for all primes $p$ and for all $k \in \mathbb{N}$. In particular, one may define a suitable action of $\left(\mathcal{A}, g_{p^{k}}\right)$ acting on $\mathcal{V}_{p^{k}}$.

Consider the algebra-action $\alpha_{p^{k}}$ of $\mathcal{A}$ in the sense of Sections 4 and 5. Now, let $\mathcal{A}_{p^{k}}$ be the quotient algebra $\mathcal{A} / \mathcal{R}_{p^{k}}$ of $\mathcal{A}$ as in (6.2), for fixed $p^{k} \in \mathbb{N}$. Define a morphism, also denoted by $\alpha_{p^{k}}$, of $\mathcal{A}_{p^{k}}$ by

$$
\alpha_{p^{k}}(f)=\left[c_{i j}\right]_{(k+1) \times(k+1)} \text { in } L T_{k+1}
$$

satisfying

$$
c_{i j}= \begin{cases}0 & \text { if } i<j \\ f(1) & \text { if } i=j \\ f\left(p^{i-j}\right) & \text { if } i>j\end{cases}
$$

for all $i, j \in\{1, \ldots, k\}$. i.e., $\alpha_{p^{k}}$ of $\mathcal{A}_{p^{k}}$ in the sense of (6.12) is identical to $\alpha_{p^{k}}$ of $\mathcal{A}$ in the sense of Section 4 "up to $\mathcal{R}_{p^{k}}$."

Then one can realize that $\alpha_{p^{k}}$ acts $\mathcal{A}_{p^{k}}$ on $\mathcal{V}_{p^{k}}$, as usual matrices acting on $\mathbb{C}^{k+1}$. Indeed, if $f_{1}, f_{2} \in \mathcal{A}_{p^{k}}$, then

$$
\alpha_{p^{k}}\left(f_{1} * f_{2}\right)=\alpha_{p^{k}}\left(f_{1}\right) \alpha_{p^{k}}\left(f_{2}\right) \text { on } \mathcal{V}_{p^{k}}
$$

by (4.4).

Theorem 6.6. The pair $\left(\mathcal{V}_{p^{k}}, \alpha_{p^{k}}\right)$ forms an indefinite-inner-product-space representation of the quotient algebra $\mathcal{A}_{p^{k}}$ of the arithmetic algebra $\mathcal{A}$.

Proof. Now, let $f \in \mathcal{A}_{p^{k}}$ and let $\alpha_{p^{k}}(f)$ be the corresponding matrix in $L T_{k+1}$. Observe that;

$$
\begin{aligned}
& \left(\alpha_{p^{k}}(f)\right)\left(\begin{array}{c}
h(1) \\
h(p) \\
\vdots \\
h\left(p^{k}\right)
\end{array}\right) \\
& =\left(\begin{array}{ccccc}
f(1) & & & & 0 \\
f(p) & f(1) & & & \\
\vdots & f(p) & f(1) & & \\
\vdots & \ddots & \ddots & \ddots & \\
f\left(p^{k}\right) & \cdots & \cdots & f(p) & f(1)
\end{array}\right)\left(\begin{array}{c}
h(1) \\
h(p) \\
\vdots \\
\vdots \\
h\left(p^{k}\right)
\end{array}\right)
\end{aligned}
$$




$$
\begin{aligned}
= & \left(\begin{array}{c}
f(1) h(1) \\
f(p) h(1)+f(1) h(p) \\
\vdots \\
\vdots \\
f\left(p^{k}\right) h(1)+f\left(p^{k-1}\right) h(p)+\ldots+f(1) h\left(p^{k}\right)
\end{array}\right) \\
= & \left(\begin{array}{c}
(f * h)(1) \\
(f * h)(p) \\
(f * h)\left(p^{2}\right) \\
\vdots \\
(f * h)\left(p^{k}\right)
\end{array}\right) \in \mathcal{V}_{p^{k}},
\end{aligned}
$$

for all $h \in \mathcal{A}$ (or, $\in \mathcal{A}_{p^{k}}$ ). Therefore, $\alpha_{p^{k}}\left(\mathcal{A}_{p^{k}}\right)$ is well-defined on $\mathcal{V}_{p^{k}}$, in the sense that: if $T \in \alpha_{p^{k}}\left(\mathcal{A}_{p^{k}}\right)$ and $v \in \mathcal{V}_{p^{k}}$, then $T(v) \in \mathcal{V}_{p^{k}}$, too.

So, by (6.13) and (6.14), $\alpha_{p^{k}}$ is a well-defined algebra-action of $\mathcal{A}_{p^{k}}$ acting on $\mathcal{V}_{p^{k}}$. Thus the pair $\left(\mathcal{V}_{p^{k}}, \alpha_{p^{k}}\right)$ forms a well-defined indefinite-inner-product-space representation of $\mathcal{A}_{p^{k}}$.

Definition 6.7. The indefinite-inner-product-space representation $\left(\mathcal{V}_{p^{k}}, \alpha_{p^{k}}\right)$ of $\mathcal{A}_{p^{k}}$ is said to be the $p^{k}$-(indefinite-inner-product-space-)representation of $\mathcal{A}_{p^{k}}$ (or of $\mathcal{A})$.

Now, let $v_{o}=(0,0, \ldots, 0,1) \in \mathcal{V}_{p^{k}}$. Indeed, one can define an arithmetic function $o_{p^{k}}$ by

for all $n \in \mathbb{N}$, and then

$$
o_{p^{k}}(n) \stackrel{\text { def }}{=}\left\{\begin{array}{lc}
1 & \text { if } n=p^{k} \\
0 & \text { otherwise }
\end{array}\right.
$$

$$
v_{o}=\left(o_{p^{k}}(1), o_{p^{k}}(p), \ldots, o_{p^{k}}\left(p^{k}\right)\right) \text { in } \mathcal{V}_{p^{k}} .
$$

Define now a morphism $\varphi_{p^{k}}: \alpha_{p^{k}}\left(\mathcal{A}_{p^{k}}\right) \rightarrow \mathbb{C}$ by

$$
\varphi_{p^{k}}(T) \stackrel{\text { def }}{=}\left[T v_{o}, v_{o}\right]_{p^{k}},
$$

for all $T \in \alpha_{p^{k}}\left(\mathcal{A}_{p^{k}}\right)$. Then it is a linear functional on $\alpha_{p^{k}}\left(\mathcal{A}_{p^{k}}\right)$, i.e., for $f_{j} \in$ $\mathcal{A}_{p^{k}}$ with $T_{j}=\alpha_{p^{k}}\left(f_{j}\right) \in \alpha_{p^{k}}\left(\mathcal{A}_{p^{k}}\right)$, and for $t_{j} \in \mathbb{C}$, for $j=1$, 2, one can get that:

$$
\begin{aligned}
\varphi_{p^{k}}\left(t_{1} T_{1}\right. & \left.+t_{2} T_{2}\right)=\left[\left(t_{1} T_{1}+t_{2} T_{2}\right)\left(v_{o}\right), v_{o}\right]_{p^{k}} \\
& =\left[\left(t_{1} T_{1}\left(v_{o}\right)+t_{2} T_{2}\left(v_{o}\right)\right), v_{o}\right]_{p^{k}} \\
& =t_{1}\left[T_{1}\left(v_{o}\right), v_{o}\right]_{p^{k}}+t_{2}\left[T_{2}\left(v_{o}\right), v_{o}\right]_{p^{k}}
\end{aligned}
$$

by the sesqui-linearity of $[,]_{p^{k}}$

$$
=t_{1} \varphi_{p^{k}}\left(T_{1}\right)+t_{2} \varphi_{p^{k}}\left(T_{2}\right)
$$

Lemma 6.8. Let $\varphi_{p^{k}}: \alpha_{p^{k}}\left(\mathcal{A}_{p^{k}}\right) \rightarrow \mathbb{C}$ be a linear functional (6.15). Then

$$
\varphi_{p^{k}}\left(\alpha_{p^{k}}(f)\right)=f\left(p^{k}\right)=g_{p^{k}}(f),
$$

in $\mathbb{C}$, for all $f \in \mathcal{A}_{p^{k}}$, where $g_{p^{k}}$ is in the sense of (5.1.1). 
Proof. Let $T=\alpha_{p^{k}}(f)$ in $\alpha_{p^{k}}\left(\mathcal{A}_{p^{k}}\right)$ acting on $\mathcal{V}_{p^{k}}$, for $f \in \mathcal{A}_{p^{k}}$. Then

$$
\begin{aligned}
& \varphi_{p^{k}}(T)=\left[T\left(v_{o}\right), v_{o}\right]_{p^{k}} \\
& =\left[\left(\begin{array}{ccccc}
f(1) & & & & 0 \\
f(p) & f(1) & & & \\
\vdots & f(p) & f(1) & & \\
\vdots & \ldots & \ddots & \ddots & \\
f\left(p^{k}\right) & \ldots & \ldots & f(p) & f(1)
\end{array}\right)\left(\begin{array}{c}
0 \\
0 \\
\vdots \\
0 \\
1
\end{array}\right),\left(\begin{array}{c}
0 \\
0 \\
\vdots \\
0 \\
1
\end{array}\right)\right]_{p^{k}} \\
& =\left[\left(f\left(p^{k}\right), 0, \ldots, 0\right),(0,0, \ldots, 0,1)\right]_{p^{k}} \\
& =\left(f\left(p^{k}\right), 0, \ldots, 0\right) \bullet(1,0, \ldots, 0) \\
& =f\left(p^{k}\right)=g_{p^{k}}(f) .
\end{aligned}
$$

The linearity of $\varphi_{p^{k}}$, one can construct a free probability space

acting on $\mathcal{V}_{p^{k}}$.

$$
\mathfrak{A}_{p^{k}} \stackrel{\text { denote }}{=}\left(\alpha_{p^{k}}\left(\mathcal{A}_{p^{k}}\right), \varphi_{p^{k}}\right)
$$

By (6.16) and (6.17), we obtain the following theorem.

Theorem 6.9. Let $\left(\mathcal{A}, g_{p^{k}}\right)$ be the $p^{k}$-free probability space in the sense of Section 5. Then it is free-homomorphic to $\mathfrak{A}_{p^{k}}=\left(\alpha_{p^{k}}\left(\mathcal{A}_{p^{k}}\right), \varphi_{p^{k}}\right)$ of (6.17). Moreover, the free probability space $\left(\mathcal{A}_{p^{k}}, g_{p^{k}}\right)$, where $g_{p^{k}}=g_{p^{k}} / \mathcal{R}_{p^{k}}$, is equivalent to $\mathfrak{A}_{p^{k}}$ (in the sense of Voiculescu). i.e.,

$$
\left(\mathcal{A}, g_{p^{k}}\right) \text { is free-homomorphic to } \mathfrak{A}_{p^{k}}
$$

and

$$
\left(\mathcal{A}_{p^{k}}, g_{p^{k}}\right) \text { is equivalent to } \mathfrak{A}_{p^{k}} .
$$

Proof. Let $\left(\mathcal{A}, g_{p^{k}}\right)$ be the $p^{k}$-free probability space of Section 5 , determined by the point-evaluation at $p^{k}$. Define now a morphism

$$
\alpha: \mathcal{A} \rightarrow \mathfrak{A}_{p^{k}}
$$

by

$$
\alpha(f) \stackrel{\text { def }}{=} \alpha_{p^{k}}(f), \text { for all } f \in \mathcal{A} .
$$

Then $\alpha$ is linear because $\alpha_{p^{k}}$ is linear, moreover, it satisfies that

$$
\begin{aligned}
\alpha\left(f_{1} * f_{2}\right) & =\alpha_{p^{k}}\left(f_{1} * f_{2}\right) \\
& =\alpha_{p^{k}}\left(f_{1}\right) \alpha_{p^{k}}\left(f_{2}\right)=\alpha\left(f_{1}\right) \alpha\left(f_{2}\right),
\end{aligned}
$$

by (4.4). Thus, it is a well-defined algebra-homomorphism from $\mathcal{A}$ to $\mathfrak{A}_{p^{k}}$. Also, it satisfies that 


$$
\varphi_{p^{k}}(\alpha(f))=g_{p^{k}}(f),
$$

by (6.16), for all $f \in \mathcal{A}$. Therefore, $\left(\mathcal{A}, g_{p^{k}}\right)$ is free-homomorphic to $\mathfrak{A}_{p^{k}}=$ $\left(\alpha_{p^{k}}\left(\mathcal{A}_{p^{k}}\right), \varphi_{p^{k}}\right)$.

Under quotient, similarly, the free probability space $\left(\mathcal{A}_{p^{k}}, g_{p^{k}}\right)$ (where $g_{p^{k}}$ is regarded as $\left.g_{p^{k}} / \mathcal{R}_{p^{k}}\right)$ is free-homomorphic to $\mathfrak{A}_{p^{k}}$, since we can get the quotient homomorphism

$$
\alpha^{\prime}=\alpha / \mathcal{R}_{p^{k}}: \mathcal{A}_{p^{k}} \rightarrow \mathfrak{A}_{p^{k}} .
$$

So, it suffices to check that $\alpha^{\prime}$ is bijective from $\mathcal{A}_{p^{k}}$ onto $\mathfrak{A}_{p^{k}}$. By the very definition of $\mathfrak{A}_{p^{k}}=\alpha_{p^{k}}\left(\mathcal{A}_{p^{k}}\right)$, the morphism $\alpha^{\prime}$ is surjective.

Now, let $f_{1} \neq f_{2}$ in $\mathcal{A}_{p^{k}}$. By the definition of the equivalence relation $\mathcal{R}_{p^{k}}$, if $f_{1}$ $\neq f_{2}$ in $\mathcal{A}_{p^{k}}$, then there exists at least one entry $j$ in $\{1, \ldots, k+1\}$, such that

$$
f_{1}\left(p^{j}\right) \neq f_{2}\left(p^{j}\right) \text { in } \mathbb{C},
$$

and hence,

$$
\alpha_{p^{k}}\left(f_{1}\right)=\alpha^{\prime}\left(f_{1}\right) \neq \alpha^{\prime}\left(f_{2}\right)=\alpha_{p^{k}}\left(f_{2}\right)
$$

in $\mathfrak{A}_{p^{k}}$. Therefore, the homomorphism $\alpha^{\prime}$ is injective, too.

So, the homomorphism $\alpha^{\prime}: \mathcal{A}_{p^{k}} \rightarrow \mathfrak{A}_{p^{k}}$ is bijective, equivalently, it is an algebraisomorphism. Therefore, two free probability spaces $\left(\mathcal{A}_{p^{k}}, g_{p^{k}}\right)$ and $\mathfrak{A}_{p^{k}}$ of $(6.17)$ are equivalent from each other.

The above theorem demonstrates that our representation $\left(\mathcal{V}_{p^{k}}, \alpha_{p^{k}}\right)$ of the (quotient) arithmetic algebra $\mathcal{A}$ (respectively $\mathcal{A}_{p^{k}}$ ) provides the same free-distributional data.

\section{Free-Inverse Chains of $\left\{\left(\mathcal{A}_{p^{k}}, g_{p^{k}}\right)\right\}_{k=1}^{\infty}$}

In this section, motivated by Section 5.3 and Section 6, we study certain chain structures of the family $\left\{\left(\mathcal{A}_{p^{k}}, g_{p^{k}}\right)\right\}_{k=1}^{\infty}$ of free probability spaces. Throughout this section, we fix a prime $p$ in $\mathbb{N}$.

By (6.18), each $p^{k}$-free probability space $\left(\mathcal{A}, g_{p^{k}}\right)$ is free-homomorphic to the free probability space

$$
\mathfrak{A}_{p^{k}}=\left(\alpha_{p^{k}}\left(\mathcal{A}_{p^{k}}\right), \varphi_{p^{k}}\right),
$$

induced by the representation $\left(\mathcal{V}_{p^{k}}, \alpha_{p^{k}}\right)$, and hence, the quotient-free probability space $\left(\mathcal{A}_{p^{k}}, g_{p^{k}}\right)$ is equivalent to $\mathfrak{A}_{p^{k}}$, for all $k \in \mathbb{N}$, where $\mathcal{A}_{p^{k}}=\mathcal{A} / \mathcal{R}_{p^{k}}$. So, without loss of generality, studying a certain chain structure of the family $\left\{\left(\mathcal{A}_{p^{k}}, g_{p^{k}}\right)\right\}_{k=1}^{\infty}$ is to investigate that of $\left\{\mathfrak{A}_{p^{k}}\right\}_{k=1}^{\infty}$.

Theorem 7.1. Let $\mathfrak{M}$ be the inductive limit algebra of $\left\{M_{n}(\mathbb{C})\right\}_{n=1}^{\infty}$, and let $\left\{\mathfrak{A}_{p^{k}}\right\}_{k=1}^{\infty}$ be the family (6.17) of free probability spaces. Then, in $\mathfrak{M}$, we have a chain

$$
\mathfrak{A}_{p} \subset \mathfrak{A}_{p^{2}} \subset \cdots \subset \mathfrak{A}_{p^{k}} \subset \cdots,
$$

of free probability spaces $\left\{\mathfrak{A}_{p_{k}}\right\}_{k=1}^{\infty}$, satisfying (7.1) and (7.2), where:

(7.1) there is a natural chain

$$
\alpha_{p}\left(\mathcal{A}_{p}\right) \subset \alpha_{p^{2}}\left(\mathcal{A}_{p^{2}}\right) \subset \cdots \subset \alpha_{p^{k}}\left(\mathcal{A}_{p^{k}}\right) \subset \cdots,
$$


of subalgebras in $\mathfrak{M}$, and,

(7.2) there exists a system (5.3.4) of natural projections $\left\{P_{k}\right\}_{k=1}^{\infty}$ of $\mathfrak{M}$, such that:

$$
\varphi_{p^{k}}\left(P_{k+1}\left(\alpha_{p^{k+1}}(f)\right)\right)=\varphi_{p^{k}}\left(\alpha_{p^{k}}(f)\right)=f\left(p^{k}\right)
$$

for all $k \in \mathbb{N}$.

Proof. It is trivial by the following commuting ladder;

$$
\begin{aligned}
& M_{2}(\mathbb{C}) \subset M_{3}(\mathbb{C}) \subset \cdots \subset M_{k+1}(\mathbb{C}) \subset \cdots \subset \mathfrak{M} \\
& \alpha_{p}\left(\mathcal{A}_{p}\right) \subset \alpha_{p^{2}}\left(\mathcal{A}_{p^{2}}\right) \subset \cdots \subset \alpha_{p^{k}}\left(\mathcal{A}_{p^{k}}\right) \subset \cdots \subset \mathfrak{M},
\end{aligned}
$$

set-theoretically and algebraically, i.e., $\mathcal{A}_{p^{k}}$ is not only a subset of both $M^{k+1}(\mathbb{C})$ and $\mathfrak{A}_{p^{k}}$, but also a subalgebra of both $M^{k+1}(\mathbb{C})$ and $\mathfrak{A}_{p^{k}}$ (under the inductive limit of Section 3), for all $k \in \mathbb{N}$.

The statement (7.2) can be proven by (5.3.3) and (6.18).

The above theorem shows that we have a certain chain of algebras whose freedistributional data is compressed by inversely.

Definition 7.2. The chain of subalgebras $\left\{\alpha_{p^{k}}\left(\mathcal{A}_{p^{k}}\right)\right\}_{k=1}^{\infty}$ in $\mathfrak{M}$, in the sense of (7.1), satisfying (7.2) is called the free-inverse chain of $\mathcal{A}$ (determined by a prime $p)$. Denote such a chain by $\mathfrak{C}_{p^{k}}$

i.e., whenever we fix a prime, then we obtain the free-inverse chain $\mathfrak{C}_{p^{k}}$ of free probability spaces $\left\{\mathfrak{A}_{p^{k}}\right\}_{k=1}^{\infty}$ in $\mathfrak{M}$.

\section{REFERENCES}

1. D. Alpay, A. Dijksma, J. van der Ploeg, and H. S. V. de Snoo,Holomorphic Operators Between Krein Spaces and the Number of Squares of Associated Kernel, Oper. Theory Adv. Appl. 59 (1992), 11-29.

2. D. Alpay, and I. Lewkowicz, An easy-to-compute factorization of rational generalized positive functions, Systems Control Lett. 59 (2010), no. 9, 517-521.

3. D. Alpay,Some remarks on reproducing kernel Krein spaces, Rocky Mountain J. Math.21 (1991), no. 4, 1189-1205.

4. D. Alpay, Some Krein Spaces of Analytic Functions and an Inverse Scattering Problem, Michigan Math. J. 34 (1987), no. 3, 349-359.

5. T. Ando,Linear Operators on Krein Spaces, Hokkaido University, Research Institute of Applied Electricity, Division of Applied Mathematics, Sapporo, 1979.

6. T. M. Apostol,Modular Functions and Dirichilet Series in Number Theory, Second edition. Graduate Texts in Mathematics, 41. Springer-Verlag, New York, 1990.

7. A.S. Besicovitch, Almost Periodic Functions, MR Number: 0068029 (16,817a), (1955) Dover Publisher.

8. A. S. Besicovitch, On Parseval's Theorem for Dirichlet Series, Proc. London Math. Soc. S2-26 (1927), no. 1, 25-34.

9. H. Bohr, Collected Mathematical Works, vol I, Dirichlet Series, The Riemann Zeta Function, vol II, Almost Periodic Functions, vol III, Almost Periodic Functions (Continued), Linear Congruences, Diophantine Approximations, Function Theory, Addition of Convex Curves, Other papers, MR Number: 0057790 (15,276i) (1952) Published by Dansk Matematisk Forening Kobenhavn. 
10. J. B. Bost, and A. Connes, Hecke Algebras, Type III-Factors and Phase Transitions with Spontaneous Symmetry Breaking in Number Theory, Selecta Math. (N.S.) 1 (1995), no. 3, 411-457.

11. D. Bump, Automorphic forms and representations, Cambridge Studies in Advanced Mathematics, 55. Cambridge University Press, Cambridge, 1997.

12. I. Cho, Classification on Arithmetic Functions and Corresponding Free-Moment LFunctions, Bulletin Korean Math. Soc. (2013), (to appear).

13. I. Cho, Operators Induced by Prime Numbers, Methods Appl. Anal. 19 (2012), no. 4, 313339.

14. I. Cho, p-adic Banach-space operators and adelic Banach-space operators, Opuscula Math. (2013), (to appear).

15. I. Cho, Free distributional data of arithmetic functions and corresponding generating functions determined by gaps between primes, Complex Anal. Oper. Theory 8 (2014), no. 2, $537-570$.

16. I. Cho and T. GillespieArithmetic functions and corresponding free probability determined by primes, (2013), Submitted to Rocky Mountain J. Math.

17. I. Cho and T. Gillespie, Real numbers acting on arithmetic functions, (2012) Submitted to INTEGERS: Elec. J. Combinat. Numb. Theo.

18. I. Cho and P.E.T. Jorgensen, Krein-space operators induced by Dirichlet characters, Special Issue: Contemp. Math. (2013) Amer. Math. Soc.

19. I. Cho and P.E.T. Jorgensen, Arithmetic functions in harmonic analysis and operator theory, (2013) Submitted to Springer Book Project: Handbook of Operator Theory.

20. G. Christner, Application of the extension properties of operators on Krein spaces, Univ. of Virginia, (1993) PhD Thesis.

21. J.L. Dalecki and M.G. Krein, Stability of solutions of differential equations in Banach space, (Translated from the Russian by S. Smith), Translations of Math. Monographs, Vol. 43. American Mathematical Society, Providence, R.I., 1974.

22. H. Davenport, Multiplicative number theory, 3-rd Ed., Grad. Texts in Math., 74. SpringerVerlag, New York, 2000.

23. M.A. Dritchel and J. Rovnyak, Operators on indefinite inner product spaces, Lecture Note, Univ. of Virginia, Dept. of Math., (1996).

24. A.J. Hildebrand, Introduction to analytic number theory, Lecture Notes, availble at http://www.math.uiuc.edu/ ${ }^{\sim}$ hilderbr/ant, (2006).

25. T. Gillespie, superposition of zeroes of automorphic L-functions and functoriality, Univ. of Iowa, (2010) PhD Thesis.

26. T. Gillespie, Prime number theorems for Rankin-Selberg L-functions over number fields, Sci. China Math. 54 (2011), no. 1, 35-46.

27. M.G. Krein and H. Langer,Uber einige Fortsetzungs probleme, die eng mit der Theorie hermitescher Operatoren im Raume $\Pi_{\kappa}$ zusammenhngen, I. Einige Funktionenklassen und ihre Darstellungen. Math. Nachr. 77 (1977), 187-236.

28. J.C. Lagarias, Euler's Constant: Euler's work and modern developments, Bulletin Amer. Math. Soc., 50 (2013), no. 4, 527-628.

29. P.D. Lax and R.S. Phillips, Scattering theory for transport Phenomena, Funct. Anal. Proc. Conf. Irvine. Calif. (1966), 119 - 130.

30. M.B. Nathanson, Additive number theory, Grad. Text in Math., 164, ISBN: 0-387-94656-X, Springer-Verlag, 1996.

31. D.J. Newman, Analytic number theory, Grad. Text in Math., 177, ISBN: 0-387-98308-2, Springer-Verlag, 1998.

32. R.S. Phillips, The extension of dual subspaces invariant under an algebra, Proc. Internat. Sympos. Linear Spaces, Jerusalem Acad. Press, (1960), 366-398.

33. F. Radulescu, Random matrices, amalgamated free products and subfactors of the $C^{*}-$ algebra of a free group of nonsingular index, Invent. Math., 115 (1994), 347-389. 
34. R. Speicher, Combinatorial theory of the free product with amalgamation and operatorvalued free probability theory, Mem. Amer. Math. Soc. 132 (1998), no. 627.

35. V.S. Vladimirov, I.V. Volovich and E.I. Zelenov,p-adic analysis and mathematical physics, Ser. Soviet \& East European Math., vol 1, ISBN: 978-981-02-0880-6, (1994) World Scientific.

36. D. Voiculescu, K. Dykemma, and A. Nica, Free random variables, CRM Monograph Series, vol 1, (1992).

St. Ambrose Univ., Dept. of Math., 421 Ambrose Hall, 518 W. Locust St., DAVENPORT, IOWA, 52803, USA.

E-mail address: choilwoo@sau.edu

Univ. of Iowa, Dept. of Math., 14 McLean Hall, Iowa City, Iowa, 52242, USA.

E-mail address: palle-jorgensen@uiowa.edu 